\title{
A histone modification-based synthetic circuit to engineer temporal gene expression in Arabidopsis floral meristems
}

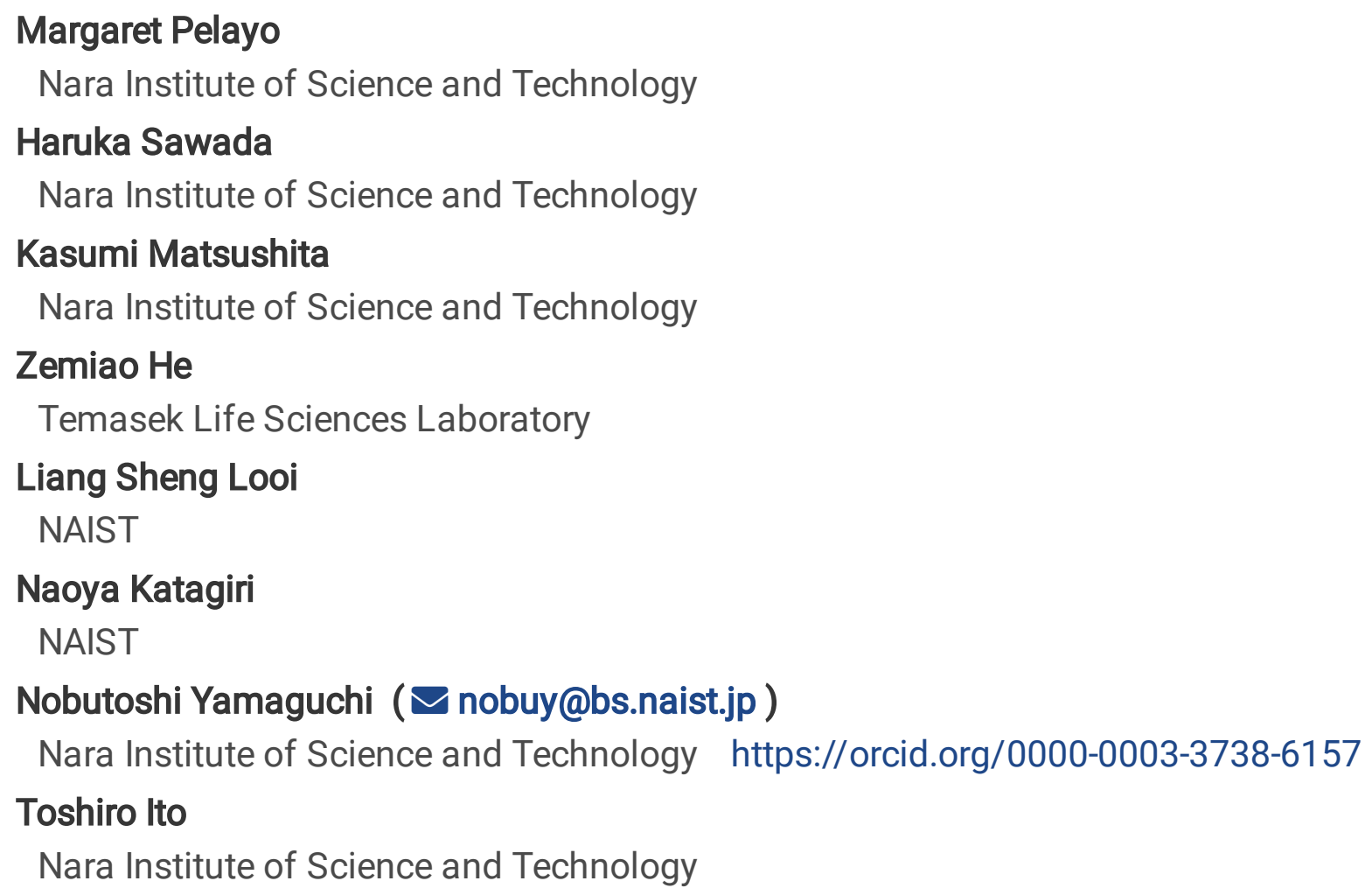

Article

Keywords:

Posted Date: January 19th, 2022

DOI: https://doi.org/10.21203/rs.3.rs-1240596/v1

License: (c) (i) This work is licensed under a Creative Commons Attribution 4.0 International License. Read Full License 
1 Title: A histone modification-based synthetic circuit to engineer temporal gene expression in Arabidopsis floral meristems

Authors: Margaret Anne Pelayo ${ }^{1}$, Haruka Sawada ${ }^{1}$, Kasumi Matsushita ${ }^{1}$, Zemiao $\mathrm{He}^{2}$, Liang Sheng Looi $^{1}$, Naoya Katagiri ${ }^{1}$, Nobutoshi Yamaguchi ${ }^{1 *}$ and Toshiro Ito ${ }^{1 *}$

6

7 Affiliations:

8 1. Division of Biological Science, Graduate School of Science and Technology, Nara Institute of 9 Science and Technology, 8916-5, Takayama, Ikoma, Nara, 630-0192, Japan

10 2. Temasek Life Sciences Laboratory, National University of Singapore, Singapore 117604, Singapore 11

*Correspondence to: nobuy@bs.naist.jp; itot@bs.naist.jp

13

14 


\section{Abstract:}

16 The termination of floral meristems is regulated by the MADS domain transcription factor 17 AGAMOUS (AG) by passively diluting the H3K27me3 mark along the KNUCKLES (KNU) coding 18 sequence. How many other downstream genes are similarly regulated by this intrinsic epigenetic timer 19 and whether it can be harnessed for engineering synthetic circuits are unknown. Here, we describe a biotimer gene regulatory network downstream of $\mathrm{AG}$ and manipulate the timing of $K N U$ expression through a synthetic system. We manipulated temporal gene expression using the del region from the $K N U$ coding sequence, which is decorated by H3K27me3-marked nucleosomes. Increasing the number of del copies delayed and reduced $K N U$ expression in a PRC2- and cell cycle-dependent manner. We propose that PRC2 deposits $\mathrm{H} 3 \mathrm{~K} 27 \mathrm{me} 3$, while cell divisions dilute H3K27me3 accumulation on the extended $K N U$ coding sequence. Our results shed light on temporal transitions governing flower development and provide a novel tool for tunable gene expression. 
Main:

Plants continuously produce new organs throughout their life via groups of undifferentiated cells called meristems whose activity is dynamically regulated to balance cell proliferation and differentiation ${ }^{1}$. In Arabidopsis (Arabidopsis thaliana), floral meristems (FMs) form a fixed number of floral organs ${ }^{2}$. At early stages, FMs proliferate to produce cells. At later stages of FM growth, the balance shifts towards cell differentiation to irreversibly terminate cell proliferation ${ }^{3-5}$. Proper timing of this developmental shift, designated FM termination, fixes the number of floral organs.

The MADS domain transcription factor AGAMOUS (AG) is the central regulator of FM termination $^{6-10}$. AG represses WUSCHEL (WUS), which maintains the stem cell population in FMs. $\mathrm{AG}$ (as a tetramer with SEPALLATA [SEP] proteins) can directly and indirectly repress WUS: directly by recruiting PRC2 to $W U S^{2}$, and indirectly via the two AG downstream targets CRABS CLAW (CRC, a YABBY transcription factor) and KNUCKLES (KNU, a $\mathrm{C}_{2} \mathrm{H}_{2}$-type zinc-finger protein). CRC regulates auxin homeostasis for proper FM termination and gynoecium formation through its targets TORNADO2 (TRN2) and YUCCA4 (YUC4), encoding a putative auxin transporter from the tetraspanin transmembrane protein family and an auxin biosynthetic enzyme, respectively ${ }^{11,12}$. AG also activates KNUCKLES $(K N U)$ encoding a transcriptional repressor of $W U S^{2,11,13-20}$. KNU transcriptional activation shows a 2-day delay after AG recruitment to the $K N U$ promoter ${ }^{14}$. At stage 2 of flower development, $K N U$ expression is initially repressed by the Polycomb repressive complex 2 (PRC2), which establishes and maintains repressive trimethylation at lysine 27 of histone $\mathrm{H} 3$ (H3K27me3) along the $K N U$ coding sequence ${ }^{14,15,21}$. By floral stage 3, AG accumulates and displaces PRC2 from the $K N U$ locus, which decreases $\mathrm{H} 3 \mathrm{~K} 27 \mathrm{me} 3$ deposition mediated by $\mathrm{PRC} 2$, thereby lowering $\mathrm{H} 3 \mathrm{~K} 27 \mathrm{me} 3$ levels in a cell cycle-dependent manner. $K N U$ transcription is activated after 2 days of cell divisions, just before FM termination ${ }^{15}$. This intrinsic timing mechanism (referred to here as a "biotimer") is essential for proper flower development, as altering $K N U$ temporal expression results in either premature FM termination or indeterminacy ${ }^{14,22}$. Although AG has about 2,000 known target genes, $K N U$ is the only one regulated by the $\mathrm{AG}$ biotimer $^{23}$. Other AG targets associated with flower development and with regulatory roles include STYLISH1 (STY1) for proper carpel development by regulating auxin biosynthesis, INDETERMINATE DOMAIN12 (IDD12) associated with gibberellic acid biosynthesis and response, the recently characterized modulator of root system architecture $A T$ HOOK MOTIF NUCLEAR LOCALIZED PROTEIN (AHL18), and PLATZ10 (PZ10), a member of the novel transcription factor class of plant-specific zinc-dependent DNA-binding proteins ${ }^{24-27}$. How these genes are regulated by AG remains largely unknown.

PRC2 and other polycomb group proteins establish repressive H3K27me3 marks at target genes that result in transient and heritable silencing by chromatin compaction ${ }^{28,29}$. In such cases, $\mathrm{H} 3 \mathrm{~K} 27 \mathrm{me} 3$ is heritable across cell divisions and yet also responds dynamically to intracellular and extracellular cues $^{30}$. In plants, PRC2 regulates developmental transitions and cell type 
specification $^{31,32}$. Arabidopsis PRC2 forms specific protein complexes with different functions over a particular developmental phase ${ }^{31-34}$. For example, flower development is largely associated with the EMF2-PRC2 variant composed of CURLY LEAF (CLF, also a homolog named SWINGER [SWN]), EMBRYONIC FLOWER 2 (EMF2), FERTILIZATION-INDEPENDENT ENDOSPERM (FIE), and MULTICOPY SUPPRESSOR OF IRA 1 (MSI1) ${ }^{32}$. Although PRC2 function has been described in detail, questions remain regarding how it drives proper temporal transitions during development. Various models have been postulated for the stable propagation of $\mathrm{H} 3 \mathrm{~K} 27 \mathrm{me} 3$ marks over generations and for their removal for activating gene expression ${ }^{35-37}$. Modes of chromatin reprogramming upon H3K27me3 removal can be controlled actively through histone demethylases, as well as passively when PRC2 is inactive at target loci ${ }^{38}$. KNU transcriptional activation relies on passive H3K27me3 removal as an integration of cell division cycles, ensuring the proper timing of $K N U$ expression and FM termination ${ }^{15}$.

The transition from floral indeterminacy to determinacy signals the onset of cell differentiation and the cessation of cell proliferation, suggesting that FM cells become committed to a differentiation pathway ${ }^{39}$. Cell differentiation programs are tightly linked to cell cycle regulation to establish tissues and organs ${ }^{40}$. Cell cycle progression is driven mainly by the interaction of cyclins (CYCs) and cyclin-dependent kinases (CDKs), a class of Ser/Thr kinases ${ }^{41}$. CYCs and CDKs form complexes that phosphorylate various target proteins at specific phases of the cell cycle, namely $\mathrm{G}_{1}$ (gap 1), S (synthesis), $\mathrm{G}_{2}$ (gap 2), and M (mitosis). Among CDKs, B-type CDKs (CDKBs) are plantspecific and are thought to be solely associated with cell cycle regulation ${ }^{41}$. CDKBs comprise two subgroups, CDKB1 and CDKB2, each represented by two members (CDKB1;1, CDKB1;2, CDKB2;1 and $\mathrm{CDKB}$;2). $C D K B 1$ expression is associated with $\mathrm{S}, \mathrm{G}_{2}$, and $\mathrm{M}$ phases while $C D K B 2$ expression is associated with $\mathrm{G}_{2}$ and $M$ phases. $C D K B$ activity is a marker for $\mathrm{G}_{2} / \mathrm{M}$ progression. The action of $\mathrm{CYC} / \mathrm{CDK}$ complexes can be regulated by $\mathrm{CYC} / \mathrm{CDK}$ inhibitors (CKIs) ${ }^{41,42}$, which may belong to one of two families: INTERACTOR/INHIBITOR OF CDK/KIP-RELATED PROTEINs (ICK/KRPs) and SIAMESE-RELATED PROTEINs (SMRs). ICK/KRPs are encoded by seven genes in Arabidopsis, KRP1 (ICK1), KRP2 (ICK2), KRP3 (ICK6), KRP4 (ICK7), KRP5 (ICK3), KRP6 (ICK4), and KRP7 (ICK5), which are distantly related to CKI p $27^{\mathrm{Kip} 1}$, a member of the mammalian Kip/Cip CKI family ${ }^{43}$. KRPs function in a dose-dependent manner and inhibit CDKA1 during the $\mathrm{G}_{1} / \mathrm{S}$ phase ${ }^{42}$. SMRs consist of 17 members; SIAMESE (SIM) was initially identified during a mutant screen for aberrant trichome formation wherein trichomes underwent cell division instead of endoreduplication ${ }^{42,44}$. KRPs inhibit entry into both $M$ and S phases, while SMRs inhibit only M phase ${ }^{42}$. How cell cycle progression affects $K N U$ transcriptional activation in the context of specific cell cycle regulators has yet to be described.

We previously showed that $d e l$, a 231-bp region in the $K N U$ coding sequence, also plays a role in proper $K N U$ temporal activation. del is a $\mathrm{H} 3 \mathrm{~K} 27$ me3-dense region bound directly by FIE whose 
deletion causes early and ectopic $K N U$ expression ${ }^{14}$, suggesting that it is required for $K N U$ repression

103 through H3K27me3 deposition. We hypothesized that extension of the $K N U$ coding sequence by adding copies of the $d e l$ region might be sufficient to repress $K N U$ expression in a cell cycle-dependent manner and increase the temporal lag to $K N U$ activation. In this study, we demonstrate that $K N U$ is a member of a biotimer gene regulatory network integrating the AG transcriptional cascade and repressive H3K27me3 status. We introduce novel candidate biotimer genes that belong to this regulatory network. We also investigated the critical mechanistic determinants of the biotimer, particularly the interplay of PRC2 activity, chromatin environment, and the cell cycle, by manipulating the $K N U$ coding sequence with del.

\section{Results}

\section{Genome-wide identification of candidate biotimer-regulated genes}

114 We defined biotimer-regulated genes as being repressed by PRC2-mediated H3K27me3 deposition 115 prior to AG recruitment before H3K27me3 removal in a cell cycle-dependent manner. We thus looked 116 for genes that show significant changes in transcript levels only at stages 6-7, are direct targets of AG 117 and PRC2, are enriched for H3K27me3, and show transcriptional delay upon AG binding associated 118 with cell division. We conducted an in silico analysis using publicly available genome-wide 119 datasets $^{23,28,45,46}$ (Fig. 1, Supplementary Table 1, Supplementary Data 1) and initially identified 11 120 fulfilling all criteria (Fig. 1a, b). Since the sporogenesis gene SPOROCYTLESS (SPL, also named 121 NOZZLE [NZZ]) is an immediate AG target induced upon $4 \mathrm{~h}$ of AG activation, unlike $K N U$, we 122 excluded $S P L$ from the final list $^{45,46}$ (Fig. 1a-c), leaving ten putative biotimer-regulated genes 123 (Supplementary Table 2).

To understand the underlying stage-specific expression changes, we performed k-means clustering on these ten genes: their transcript levels at stages 6-7 sorted them into two clusters as a function of the direction of regulation (Fig. 1c). Only two genes were upregulated, SHOOTMERISTEMLESS (STM) and ZINC FINGER PROTEIN 1 (ZPl) $)^{47,48}$, suggesting that most candidate biotimer genes remain transcriptionally repressed without functional AG by the persistence of H3K27me3 marks. Aside from AT4G16540 and AT3G11150 (encoding a heat shock protein, HEATSHOCK PROTEIN 20 (HSP20) and an oxygenase, respectively), the downregulated genes encoded either transcription factors or proteins associated with DNA-binding transcription factor activity ${ }^{49-51}$ : the zinc-finger proteins KNU, IDD12, and STY1 13,24,52; the transcription factor PLATZ10; a homeodomain-like protein (encoded by AT1G14600); and the AT hook domain-containing protein AHL1 $8^{26,52-54}$. Gene Ontology (GO) term enrichment analysis of these ten putative biotimer-regulated genes ${ }^{55}$ revealed that the top ten GO terms are mainly associated with transcription, RNA biosynthesis and metabolism within the category of cellular processes, and various cellular biosynthetic processes (Fig. 1d). Refinement of GO terms indicated regulatory roles in transcription and development ${ }^{56}$ (Fig. 
$1 \mathrm{e})$.

To understand the mechanistic determinants of the biotimer, specifically how AG, PRC2, and H3K27me3 interactions affect chromatin landscape and transcriptional activation, we compared the AG, PRC2, and $\mathrm{H} 3 \mathrm{~K} 27 \mathrm{me} 3$ binding patterns along the chromatin of selected candidate genes: $A H L 18, I D D 12$, and $S T Y 1$, along with $K N U$ as a control (Fig. 1f). As previously reported, AG directly bound to the 5' region of the $K N U$ promoter, while we observed PRC2 binding and H3K27me3 deposition along the $K N U$ gene body (Fig. 1f). We also confirmed that AG binds upstream of the coding regions of AHL18, IDD12, and STY1 (Fig. 1f). We detected substantial PRC2 binding and H3K27me3 signals in their gene bodies as well (Fig. 1f).

\section{Biotimer-dependent gene expression, PRC2 binding, and histone modifications}

149 To further explore the mechanisms of biotimer regulation, we performed reverse transcriptionquantitative PCR (RT-qPCR) for selected candidate genes during a time course using a transgenic line expressing a construct encoding the glucocorticoid receptor (GR) fused to AG in the ag- 1 mutant background, ag-1 35S::AG-GR ${ }^{45}$ (Fig. 2a-f). We collected samples over 7 days from the addition of dexamethasone (DEX) and determined relative expression levels for $K N U, I D D 12, A H L 18, P Z 10$, STY1, HD, and HSP20. KNU expression showed an upregulation 3 days after DEX treatment, in agreement with the transcriptional delay characteristic of $K N U$ activation (Fig. 2a). By contrast, we observed rapid activation of $S P L$ within 1 day after DEX treatment, as reported previously (Extended Data Fig. 1), validating our experimental set-up. Using the same RNA samples, we noticed transcriptional delays for IDD12, AHL18, and PZ10 3 days after treatment (Fig. 2b-d) and for STY1 7 days after treatment (Fig. 2e, f). No change in HSP20 expression was observed after DEX treatment

160 (Extended Data Fig. 1). These results indicated that activation of IDD12, AHL18, and PZ10 shows the most similarity to that of $K N U$, with a transcriptional delay of 1-2 days upon AG induction.

To examine whether this transcriptional delay was caused by the epigenetic biotimer, we examined the timing of removal for FIE and H3K27me3 from chromatin following AG induction during flower development by chromatin immunoprecipitation (ChIP) followed by qPCR (ChIPqPCR). We employed a floral synchronization system using the ap 1 cal $35 S:: A P 1-G R$ transgenic line, which allows the isolation of many floral buds ${ }^{55}$ and thus facilitates the analysis of PRC2 and histone deposition at distinct stages of flower development. The PRC2 component FIE bound to the gene bodies of $K N U$, IDD12, and AHL18 prior to nuclear translocation of the AP1-GR fusion protein upon

169 DEX treatment (in floral primordia at stage 1-2) (Fig. 2g). We observed a rapid reduction in FIE signals within 1 day at all three loci (Fig. 2g). After 3 days of DEX treatment, FIE signals at $K N U$, IDD12, and AHL18 reached the same background levels seen for the negative control TA3 (Fig. 2g). H3K27me3 accumulated to high levels at the KNU, IDD12, and AHL18 gene bodies before AP1 activation (in floral primordia at stage 1-2) (Fig. 2h). H3K27me3 levels at these three genes gradually 
decreased after AP1 activation. The reduction in $\mathrm{H} 3 \mathrm{~K} 27 \mathrm{me} 3$ levels between 1 day and 3 days after DEX treatment was significant (by stage 6 of flower development) (Fig. 2h).

Genes regulated by the epigenetic biotimer should display a passive dilution of H3K27me3 in a cell cycle-dependent manner ${ }^{57,58}$. To test for this, we exposed plants to the cell cycle inhibitor olomoucine (olo). After 3 days of DEX and olo treatment, reduction of CDKB1;1 was observed (Extended Data Fig. 2). Under this condition, $K N U$, IDD12, and $A H L 18$ were induced in DEX-treated ag-1 35S::AG-GR (Fig. 2a-c, i), but olo treatment compromised the extent of this induction. Taken together, these findings suggest that $K N U, I D D 12$ and $A H L 18$ are regulated by the AG-mediated epigenetic biotimer.

\section{Extra $K N U$ del copies delay $K N U$ temporal activation and reduce $K N U$ expression}

185 We previously observed that deleting a 231-bp region ( $\mathrm{del}$ ) leads to precocious and ectopic $K N U$ 186 expression ${ }^{14}$. Thus, we hypothesized that providing more copies of this sequence would increase repressive H3K27me3 marks and further delay the induction of $K N U$ during floral development. To examine this possibility, we introduced one to six copies of the del region (1del-6del) into the $p K N U:: K N U$-GUS construct (Fig. 3a) and classified the resulting GUS signal as weak, intermediate, or strong in the generated primary transformants $\left(\mathrm{T}_{1}\right)$ at floral stages 6-8 (Extended Data Fig. 3). We defined a signal as weak in the absence of GUS staining at stage 6; as intermediate with minimal signal detected at stage 6; and as strong with clearly detected GUS signal at stages 5-6. We obtained the highest occurrence of strong GUS signals for the $p K N U:: K N U-G U S$ reporter with 1del, while the frequency of strong GUS signals decreased as the number of del copies increased (Fig. 3b). The lower GUS signal was not an indirect result of the longer coding region of the construct, as replacing $3 d e l$ or 6del with DNA fragments of approximate equivalent lengths (one or two copies of the cyan fluorescent protein $[C F P]$ coding sequence) produced similar $\mathrm{T}_{1}$ GUS signal intensities as $p K N U:: K N U-G U S$ (Extended Data Fig. 4), suggesting that the change in GUS accumulation depends on the presence of $d e l$. We characterized the GUS staining pattern in representative $p K N U:: K N U$-GUS, $3 \mathrm{del}$, and $6 \mathrm{del}$ homozygous lines during flower development (Fig. 3c-q). pKNU::KNU-GUS expression started from floral stage 5, with a moderate GUS signal (Fig. 3c, d). This expression rose to a strong GUS signal at stage 6 (Fig. 3e) and later decreased to a moderate signal from stage 7 to stage 8 (Fig. 3f, g). This expression pattern followed the timing of $K N U$ mRNA accumulation in wildtype (WT) plants ${ }^{13}$. Not only timing of $3 \mathrm{del} / 6 \mathrm{del}$ activation, but also their peaks were shifted. With the 3 del construct, we detected only a faint GUS signal at the center of the FM at stage 5 (Fig. 3h, i) that persisted into stage 6 before increasing to a moderate signal at stage 7 and returning to a weak signal at stage 8 (Fig. 3j-1). Expression timing was further delayed in 6 del. Unlike, $p K N U:: K N U$-GUS or $3 \mathrm{del}, 6 \mathrm{del}$ was not expressed at stage 5. $6 \mathrm{del}$ also exhibited a weak GUS signal initially at stage 6 until 
211 Based on RT-qPCR analysis, GUS transcript levels were substantially lower in 6del lines compared to

$212 p K N U:: K N U$-GUS lines (Fig. 3r), while endogenous $K N U$ mRNA levels were comparable (Extended

213 Data Fig. 5). Furthermore, GUS activity was much lower in 6 del lines relative to $p K N U:: K N U-G U S$ 214 lines (Fig. 3s). These results demonstrate that adding more copies of del to the $K N U$ coding sequence 215 can extend the delay of and reduce $K N U$ expression.

216 To investigate the trimethylation status of H3K27 in $p K N U:: K N U-G U S$ and 6 del lines, we 217 performed H3K27me3 ChIP-qPCR, which showed an enrichment for H3K27me3 levels in 6del lines 218 compared to $p K N U:: K N U$-GUS lines (Fig. 3t). However, H3 levels were comparable between the lines, 219 as evidenced by H3 ChIP (Extended Data Fig. 6), indicating that the observed difference is due to 220 changes in the deposition of H3K27me3 marks. These results suggest that the delay in $K N U$ 221 transcriptional activation is due to increased deposition of repressive H3K27me3 with more copies of the del sequence.

\section{PRC2 deposits H3K27me3 on the extended $K N U$ coding sequence}

We first detected $6 \mathrm{del}$ expression in WT at stage 6 of floral development, later than for pKNU::KNU-GUS and constructs with fewer del copies (Fig. 3). To test whether PRC2 was responsible for $\mathrm{H} 3 \mathrm{~K} 27 \mathrm{me} 3$ deposition on the extended $K N U$ coding sequence, we investigated the contribution of the PRC2 components FIE and CLF to 6 del H3K27me3 deposition (Fig. 4a-q). Since loss-of-function mutants in FIE are embryonic lethal, we used the co-suppression lines $35 S: \because G F P$-FIE (hereafter referred to as fie) with reduced FIE levels as an alternative ${ }^{59}$. We also crossed the $6 \mathrm{del}$ construct into the mutant $c l f-28(c l f)$. In WT, we failed to detect GUS signal from the 6 del construct at stages 4 and 5 (Fig. 4a, b). GUS signal then appeared at stage 6 and remained weak until stage 7 (Fig. 4c, d), with little to no GUS signal at stage 8 (Fig. 4e). Although GUS signal was absent from the fie and $c l f$ mutants at stage 4 , the 6 del construct showed precocious expression at stage 5 (Fig. 4a, b, f, g, k, 1), with rising GUS signal intensity at stages 6 and 7 (Fig. 4c, d, h, i, m, n) before declining by stage 8 in $c l f$ (Fig. 4e, o). We hypothesize that the lower GUS signals obtained in $c l f$ reflect the weaker phenotypes seen with $C L F$ loss of function ${ }^{60,61}$. We detected GUS signal at stage 8 in the fie background (Fig. 4j). GUS transcript accumulation was then quantified for fie relative to 6del WT. Higher GUS signal correlated with higher GUS transcript levels in fie compared to 6del WT, as determined by RT-qPCR (Fig. 4p). We also investigated H3K27me3 levels in the fie background by ChIP-qPCR. We determined that H3K27me3 levels along the 6del transgene are substantially lower in fie and clf compared to WT (Fig. 4q). We conclude that the increase in GUS expression is due to a disruption in the PRC2 machinery, attenuating H3K27me3 deposition at target loci.

\section{The cell cycle regulates $\mathrm{H} 3 \mathrm{~K} 27 \mathrm{me} 3$ dilution on the extended $K N U$ coding sequence}


The cell cycle plays an important role in $K N U$ biotimer regulation by diluting $\mathrm{H} 3 \mathrm{~K} 27 \mathrm{me} 3$ along the $K N U$ coding sequence. Arabidopsis grows optimally in temperatures of $12-27^{\circ} \mathrm{C}$ but can respond to changes in ambient temperature by adjusting growth rates, with lower temperatures slowing cellular processes including the cell cycle ${ }^{62,63}$. We measured transcript levels for the selected CKI genes KRP1, KRP2, SMR1, SMR2, and SMR3 in 6 del lines in the WT background grown at $22^{\circ} \mathrm{C}$ or $18^{\circ} \mathrm{C}$. We determined that $C K I$ expression is induced at $18^{\circ} \mathrm{C}$ relative to $22^{\circ} \mathrm{C}$, suggesting that the cell cycle is inhibited (Fig. 5a). We also explored the stage-specific accumulation of the core cell cycle and $\mathrm{G}_{2} / \mathrm{M}$ transition regulator CYCB1 as a fusion protein to yellow fluorescent protein (YFP) at $22^{\circ} \mathrm{C}$ and $18^{\circ} \mathrm{C}$ (Fig. 5b-e). We detected a prevalent CYCB1-YFP signal at stages 4 and 6 at $22^{\circ} \mathrm{C}$ that is much weaker in plants grown at $18^{\circ} \mathrm{C}$, suggesting that cyclin activity is blocked by lower temperature conditions, presumably due to higher CKI activity (Fig. 5b-e).

We characterized the consequences of a slower cell cycle on 6 del activation by growing plants at $18^{\circ} \mathrm{C}$. We failed to detect GUS signal from the $6 \mathrm{del}$ construct during stages 4-6 and only observed GUS signal at stage 7, which returned to below detection limits at stage 8 (Fig. $5 \mathrm{f}-\mathrm{o}$ ). H3K27me3 levels showed an inverse correlation with GUS signal, with higher levels along the 6del construct at $18^{\circ} \mathrm{C}$ compared to $22^{\circ} \mathrm{C}$ (Fig. 5v). These results suggest that lower temperatures further delay $K N U$ activation in 6 del, likely due to the slower dilution of $\mathrm{H} 3 \mathrm{~K} 27 \mathrm{me} 3$ marks along the extended $K N U$ region.

To further investigate whether mutations in cell cycle genes affected $K N U$ activation, we crossed the $6 \mathrm{del}$ construct into a quintuple krp1 krp2 krp3 krp4 krp7 mutant (referred to as $k r p-q)^{64}$ (Fig. $5 \mathrm{p}-\mathrm{t}$ ). The $k r p-q$ mutant exhibits increased cell proliferation and leaf cell size, likely as a result of enhanced CDK activity leading to greater levels of phosphorylated RETINOBLASTOMARELATED 1 (RBR1) and upregulation of the E2F pathway ${ }^{64}$. As with $p r c 2$ mutants, the $k r p-q$ mutant also showed precocious GUS signal derived from the $6 \mathrm{del}$ construct from stage 5 floral buds (Fig. $5 \mathrm{f}$, $\mathrm{g}, \mathrm{p}, \mathrm{q}$ ) that reached a peak at stages 6 and 7 (Fig. 5r, s). GUS transcript levels were higher in krp-q compared to WT (Fig. 5g-i, q-s). Although 6 del in WT produced only a weak signal, $k r p-q$ mutants still possessed moderate GUS signal derived from 6 del at stage 8 (Fig. $5 \mathrm{j}, \mathrm{t}$ ). In addition, GUS transcripts accumulated to about 2.5-fold higher levels in krp-q relative to WT (Fig. 5u), which was correlated with lower H3K27me3 levels along the del sequence in krp-q compared to WT (Fig. 5t). These results suggest that enhancing cell cycle activity by removing CDK inhibition results in accelerated H3K27me3 dilution along del.

To examine the effects of raising cell cycle activity on $K N U$ and $6 d e l$ activation, we used the $35 S:: C Y C D 3 ; 1$ line (Extended Data Fig. 7), which has an extended $\mathrm{G}_{2}$ phase due to delayed activation of cyclin $\mathrm{B}$ and $\mathrm{G}_{2} / \mathrm{M}$ gene expression ${ }^{65}$. We observed a delayed activation of $K N U$ expression in $35 S:: C Y C D 3 ; 1$, as revealed by the $p K N U:: K N U-G U S$ and 6 del constructs (Extended 
WT, we detected no GUS signal and a faint signal in the 35S::CYCD3;1 background at stages 6 and 7, respectively (Extended Data Fig. 7k-t). This result suggests that the cell cycle regulates H3K27me3 dilution along the extended $K N U$ coding sequence.

\section{Discussion}

\section{Temporal control of gene expression by a biotimer for plant development}

288 In this study, we demonstrated that $K N U$ is part of a biotimer gene regulatory network that integrates 289 AG transcriptional signaling and $\mathrm{H} 3 \mathrm{~K} 27 \mathrm{me} 3$ status. We identified ten candidate biotimer genes 290 (including $K N U$ ) that are direct downstream AG targets, are highly enriched for H3K27me3 along the 291 gene body, and show delayed transcriptional activation upon AG binding associated with cell division 292 cycles. This mode of transcriptional regulation may be recurring throughout flower development, 293 especially during FM termination. We confirmed PRC2 eviction and subsequent cell cycle-dependent 294 H3K27me3 dilution at the candidate biotimer loci IDD 12 and AHL18 after AG activation, as observed 295 with $K N U$ regulation (Fig. 6). IDD12 encodes a member of the IDD family of transcription factors 296 involved in radial root patterning ${ }^{20,72-74}$. The regulation of IDD12 via biotimer may therefore play an 297 important role in the establishment and propagation of asymmetric cell divisions, and thus cellular 298 patterns, in FMs. AHL18 is a regulator of lateral root development ${ }^{26}$. AHL family members have been 299 implicated in the initiation of leaf senescence, hypocotyl elongation, phytohormone biosynthesis, and 300 flowering time regulation ${ }^{68-75}$. AHL21, also named GIANT KILLER, is a regulator of reproductive 301 organ specification during flower development, whose encoding gene is expressed under direct AG 302 control $^{69}$. AHLs have multilevel modes of regulation, linking transcription factor activity with 303 chromatin remodeling, and enabling the activation and repression of genes simultaneously ${ }^{69,75}$. Precise 304 control of chromatin remodeling downstream of a biotimer may be important for FM termination 305 and/or flower development. As IDD12 and AHL18 are members of transcription factor families 306 participating in root development, a wider set of genes may also be regulated throughout other phases 307 of plant development. Functional analysis of these genes will be critical to dissecting their precise roles during flower development.

\section{Engineering a time lag mechanism by the addition of $\mathrm{H3K27me3-bound} \mathrm{regions}$}

311 We investigated the mechanistic determinants of the biotimer by manipulating the number of del 312 sequences within the KNU locus driving the expression of a GUS reporter system. More copies of del 313 resulted in a progressive delay of $K N U$ temporal activation (Fig. 6). Using the $p K N U:: K N U-G U S$ 314 reporter, we confirmed that the GUS staining pattern parallels that of endogenous $K N U$ expression, 315 with staining during stages 4-8 (Fig. 3c-g), in agreement with the typical time delay observed for $316 K N U$ expression after AG activation begins at floral stage $3^{15}$. The $K N U$ coding sequence is $451 \mathrm{bp}$, 317 equivalent to approximately three nucleosomes of $\sim 150-200$ bp of DNA each wrapped around the 
histone octamer and each marked with $\mathrm{H} 3 \mathrm{~K} 27 \mathrm{me} 3$. The del region from the second half of the $K N U$ coding sequence consists of $231 \mathrm{bp}$, roughly corresponding to one nucleosome, and is bound by FIE and decorated by H3K27me3 marks. Each cell division cycle from floral stage 3 onward may result in the removal of $\mathrm{H} 3 \mathrm{~K} 27 \mathrm{me} 3$ on a marked nucleosome at $K N U$, suggesting that stage 4 proliferating cells may be a mixture of cells with one nucleosome without H3K27me3 (initial cells) or with two unmarked nucleosomes (cell progeny cells). We also hypothesize that the H3K27me3 marks diluted during cell division reside along the $\mathrm{del}$ region, due to the demonstrated role of this region in regulating $K N U$ temporal activation. By stage 5, H3K27me3 marks may thus be completely removed in some cells, which start to exhibit $K N U$ expression. By stage 6, $K N U$ expression reaches its peaks as most proliferating cells are now unmarked by H3K27me3. This time frame corresponds to the proper timing of FM termination to ensure the development of proper numbers of floral organs. By stage 7 and until stage 8, $K N U$ expression decreases (Fig. 3) Whether $K N U$ repression is also modulated by the cell cycle, methylases or a combination of both after stage 8 remains to be demonstrated.

When using $6 \mathrm{del}$, we observed a prolonged delay in $K N U$ activation (Fig. 6), with the first signs of transcriptional activation at stage 6 instead of stage 5 for $1 \mathrm{del}$. We propose that this prolonged delay is due to a greater number of nucleosomes marked with $\mathrm{H} 3 \mathrm{~K} 27 \mathrm{me} 3$ occupying the $K N U$ locus, leading to a partial dilution of $\mathrm{H} 3 \mathrm{~K} 27 \mathrm{me} 3$ marks by stage 5. Based on this model, H3K27me3 removal would still be incomplete by stage 6 in most cells, with the minimal GUS signal observed at this stage likely originating from a small pool of dividing cells wherein the H3K27me3 and FIE have been removed from the del copies. At stage 7, $\mathrm{H} 3 \mathrm{~K} 27 \mathrm{me} 3$ removal may still be incomplete in dividing cells, explaining the reduced GUS signal detected in $6 \mathrm{del}$ peaks compared to the peak $K N U$ expression in WT at stage 6. KNU repression by stage 7 may also contribute to this reduction. By stage $8, K N U$ expression is undetectable in $6 \mathrm{del}$, suggesting that the additional $\mathrm{del}$ regions augment $K N U$ repression via H3K27me3 deposition. H3K27me3 deposition on the extended $K N U$ region appears to be modulated by the PRC2 complex, based on the earlier and stronger $K N U$ induction of 6 del in the fie and $c l f$ backgrounds. An intact PRC2 machinery is thus necessary for proper $K N U$ temporal activation.

As H3K27me3 removal along $K N U$ is cell division-dependent, more cell cycles are needed to completely dilute $\mathrm{H} 3 \mathrm{~K} 27 \mathrm{me} 3$ marks along the extended region in $6 \mathrm{del}$, contributing to the observed delay and reduction in $K N U$ expression. In agreement with this notion, we observed an earlier and stronger KNU induction from the $6 \mathrm{del}$ construct in the krp-q mutant background, indicating that proper cell cycle progression is necessary for $K N U$ temporal activation by facilitating $\mathrm{H} 3 \mathrm{~K} 27$ me 3 removal. We also observed a delay in $K N U$ activation by growing plants at $18^{\circ} \mathrm{C}$, likely due to slower growth kinetics. This observation emphasizes the dynamic regulation of H3K27me3 in response to extracellular and intracellular cues and suggests a role for the cell cycle-dependent biotimer in coordinating the balance between cell proliferation and differentiation. Additional experiments will be necessary to clarify the effect of lower temperature conditions on the cell cycle and on biotimer 
regulation.

Overall, our findings highlight the complex and dynamic nature of $\mathrm{H} 3 \mathrm{~K} 27 \mathrm{me} 3$ regulation for transcriptional activation in the context of FM termination. We identified novel candidate biotimer

357 genes and proposed a mechanistic perspective of the biotimer regulation modulated by PRC2 activity,

358 chromatin environment, and the cell cycle. Future work will focus on modulating biotimer regulation 359 based on targets other than $K N U$ by investigating the minimal sequences marked with H3K27me3. 


\section{Materials and methods}

\section{Plant material and growth conditions}

The Arabidopsis accessions Landsberg erecta (Ler) or Columbia (Col-0) were used in this study. The ap1 cal 35S::AP1-GR, ap1 cal FIE-VENUS 35S::AP1-GR, ag-1 35S::AG-GR, $p C Y C B 1 ; 2: \because C Y C B 1 ; 2-Y F P$ and $35 S:: C Y C D 3$ were described previously ${ }^{15,45,65,76,77}$. pKNU::KNU$G U S^{14}, p K N U: \because \triangle K N U-G U S^{14}$ and the del series, as well as the $C F P$ and $2 x C F P$ controls, were in Ler background. The PRC2 and cell cycle mutants used for crossing with $p K N U:: K N U-G U S$ and $6 d e l$ were in Col-0. The 35S::FIE-GFP line was described previously ${ }^{59}$. clf-28 is a SALK T-DNA mutant line (SALK_139371) ${ }^{60}$, while krp1 krp2 krp3 krp4 krp7 was previously generated by crossing single SALK and GABI-kat lines (SALK_026391, SALK_130744, GABI-kat 185C07, SALK_102417, and GABI-kat 841D12, respectively) ${ }^{64}$. Seeds were sown in pots containing a mixture of soil and vermiculite $(1: 2, \mathrm{w} / \mathrm{w})$ supplemented with Hyponex (1/1000) upon germination. Sown seeds were stratified at $4{ }^{\circ} \mathrm{C}$ in the dark for 3-4 days before being transferred to long-day (16-h light/8-h darkness) conditions at $22^{\circ} \mathrm{C}$, or kept at $18^{\circ} \mathrm{C}$ in constant light. Primers used for genotyping are listed in Supplementary Table 3.

\section{Chemical treatments}

For ap1 cal 35S::AP1-GR, ap1 cal FIE-VENUS 35S::AP1-GR, and ag-1 35S::AG-GR chemical treatments, a working concentration of $100 \mu \mathrm{M}$ DEX aqueous solution was used with $0.015 \%$ (v/v) Silwet L-77. Plants used as negative controls were not treated.

For the oloumoucine (olo) treatment, ag-1 35S::AG-GR plants (no more than $8 \mathrm{~cm}$ in height) were cut at the base of the stem upon bolting and placed into 1/2 MS solution in $50 \mathrm{ml}$ Falcon tubes for 3 days under long day conditions at $22^{\circ} \mathrm{C}$. $10 \mathrm{uM}$ dexamethasone MS solution was used for the control treatment while the olo treatment contained 10uM DEX and 750uM olo. Inflorescences were collected on the third day and were trimmed to contain floral buds up to stage 12 before flash freezing in liquid nitrogen.

\section{In silico analysis}

Transcriptome data from Ó’Maoiléidigh et al. (2013) was filtered by setting the $p$ value to $<0.05$ and selecting the resulting genes from stages $3-4$ and stages $6-7$. The corresponding AG binding data was sorted by setting the $q$ value to $<1.0 \times 10^{-4}$. The dataset for PRC2 $\operatorname{targets}^{28}$ was filtered by selecting for the presence of CLF, EMF2, and FIE binding sites (features 1, 2, and 3, respectively). Each filtered dataset was then uploaded onto a web-based custom Venn diagram drawing tool (http:/bioinformatics.psb.ugent.be/webtools/Venn/) to create the Venn diagram. The heatmap was generated using WebMeV (http://mev.tm4.org). The browser views of ChIP sequencing signals for AG and $\mathrm{PRC} 2$ binding sites and $\mathrm{H} 3 \mathrm{~K} 27 \mathrm{me} 3$ were generated using $\mathrm{IGV}^{78}$. GO term analysis and 
visualization was performed using agriGO v2.0 (http://systemsbiology.cau.edu.cn/agriGOv2/) followed by GO term refinement using REVIGO (http://revigo.irb.hr/).

\section{Plasmid construction and plant transformation}

To generate the $K N U$ reporter with tandem repeats, site-directed mutagenesis was performed to insert BglII and BamHI restriction sites at positions +594 and +1 (with the translation start site counting as +1) before the GUS reporter gene to create $p K N U:: K N U-G U S(B g l \mathrm{II} / B a m \mathrm{HI})$. The $K N U$ tandem repeat includes a partial $K N U$ coding sequence from nucleotides +367 to +594 (1del); the $2 \times$ and $3 \times$ repeats were synthesized by GenScript Services (www.genscript.com). The synthesized $2 \times$ and $3 \times$ repeats were digested with $B g l \mathrm{II}$ and BamHI. The $p K N U:: K N U-G U S(B g l \mathrm{II} / B a m \mathrm{HI})$ construct was digested in a similar fashion. Subsequently, ligation was performed to create constructs carrying different numbers of tandem repeats, $2 \mathrm{del}-6 \mathrm{del}$. These constructs were recombined into Gateway vectors (Thermo Fisher Scientific) by LR recombination according to the manufacturer's instructions (ATMI). Primers used for cloning are listed in Supplementary Table 3.

To create $g K N U$-CFP-GUS and $g K N U-2 x C F P$-GUS constructs, $1 x E C F P$ and $2 x E C F P$ were amplified from pMD20-2XECFP. The resulting PCR fragments were then inserted into pCR8pKNU::KNU-GUS ${ }^{14}$ via a two-step omega-PCR strategy as described ${ }^{79}$ to create pCR8pKNU::KNU-1XECFP-GUS and pCR8-Pknu::KNU-2XECFP-GUS. Next, the vectors were introduced into the CD3-694 destination vector (or pEarleyGate303) via LR reaction (Thermo Fisher Scientific). All resulting constructs were transformed into the Ler background via the floral dip method using GV3101 strain as described previously ${ }^{80}$.

\section{GUS staining}

The primary floral bud cluster from the main inflorescence shoot of plants $\sim 3-10 \mathrm{~cm}$ in height was collected and fixed in ice-cold $90 \%(\mathrm{v} / \mathrm{v})$ acetone for $20 \mathrm{~min}$. The samples were then rinsed two to three times with Milli-Q $\mathrm{H}_{2} \mathrm{O}$ then washed two to three times with GUS buffer (without 5bromo-4-chloro-3-indolyl- $\beta$-D-glucuronide [X-Gluc]). The tissues were then infiltrated with GUS staining solution (with X-Gluc) under vacuum for $30-60 \mathrm{~s}$ and incubated at $37^{\circ} \mathrm{C}$ for $16 \mathrm{~h}$. After incubation, the staining solution was replaced with $70 \%(\mathrm{v} / \mathrm{v})$ ethanol two to three times until the tissues cleared (approximately 1 week at room temperature).

\section{4-methylumbelliferyl B-D-glucuronide assay}

To quantitatively measure GUS activity, fluorescence measurements were taken of 4methylumbelliferyl ß-D-glucuronide (4-MUG) upon conversion to 4-methyl umbelliferone (4-MU) in the presence of GUS. Up to ten primary inflorescences containing floral buds until stage 12 were collected per biological replicate and flash-frozen in liquid nitrogen. The procedure described by 
Weigel et al.$^{80}$ was followed with minor modifications. For protein extraction, the tissues were crushed in GUS extraction buffer on ice. After centrifugation, the supernatants were stored at $-80^{\circ} \mathrm{C}$ until use. An aliquot was also set aside to measure protein concentration with Qubit 4 (Thermo Fisher Scientific). For the MUG assay, the extract was added to extraction buffer mixed with $1 \mathrm{mM}$ 4-MUG, hereafter referred to as the reaction mix. Half of the reaction mix was taken out immediately and stopped by the addition of $0.2 \mathrm{M} \mathrm{Na}_{2} \mathrm{CO}_{3}$ and designated as the 0 - $\mathrm{h}$ reaction. The remaining reaction mix was incubated at $37^{\circ} \mathrm{C}$ for $1 \mathrm{~h}$. After incubation, the reaction mix was added to the stop buffer and designated as the 1-h reaction. The 0 - $\mathrm{h}$ and $1-\mathrm{h}$ reaction mixes were dispensed in triplicate in $96-\mathrm{well}$ plates and fluorescence measurements were taken with a TriStar ${ }^{2}$ LB942 microplate reader (Berthold Technologies). Excitation and emission were at $355 \mathrm{~nm}$ and $460 \mathrm{~nm}$, respectively. A 4-MU dilution series was prepared to create a 4-MU fluorescence standard curve to determine GUS activity from all samples as the amount of substrate converted to 4-MU relative to the starting protein concentration, reaction volume, and reaction duration.

\section{Sectioning}

Following GUS staining, plant tissues were dehydrated in a graded ethanol series $(80 \%[\mathrm{v} / \mathrm{v}]$, 90\%, 95\%, and 100\%) and embedded in Technovit 7100 resin (Heraeus). Tissue sections (10- $\mu \mathrm{m})$ were produced using a RM2255 microtome (Leica). The sections were dried on a $42^{\circ} \mathrm{C}$ hot plate (Sakura Finetek) then stained with $0.05 \%(\mathrm{w} / \mathrm{v})$ neutral red and mounted on a microscope slide with $500 \mu \mathrm{L}$ MountQuick (Daido Sangyo). An Axio Scope A1 microscope (ZEISS) was used to observe the sections of three or more floral buds per flower developmental stage.

\section{RT-qPCR}

Total RNA was extracted from primary floral bud clusters of the main inflorescence shoot (up to ten per biological replicate), collected and ground in liquid nitrogen. RNA extraction and cleanup was performed using the RNeasy kit (Qiagen) with an in-column DNase digest. PrimeScript RT Master Mix system (Takara) was used for first-strand cDNA synthesis. The subsequent qPCR reaction mix was prepared using the FastStart Essential DNA Green Master (Roche). qPCR was performed with a Light Cycler 480 (Roche) using the Light Cycler 480 release 1.5.1.62 SP software (Roche). Relative transcript abundance across three biological replicates was calculated using the comparative CT method; statistical analyses were performed using a two-tailed Student's $t$-test. The sequences of primer pairs used for genes of interest and the reference gene TUBULIN2 are listed in Supplementary Table 3.

\section{ChIP}

To quantify H3K27me3 and H3 enrichment, the ChIP protocol described by Yamaguchi et 
al. ${ }^{81}$ was followed with minor modifications. Primary inflorescences $(300-600 \mathrm{mg}$ ) were fixed with $1 \%(\mathrm{w} / \mathrm{v})$ formaldehyde for $15 \mathrm{~min}$, flash-frozen with liquid nitrogen and stored at $-80^{\circ} \mathrm{C}$ until use. Chromatin was extracted by nuclei isolation and sonicated to yield 500-bp fragments. Protein A beads (Thermo Fisher Scientific) were used for pre-clearing the chromatin by incubation for $1 \mathrm{~h}$ at $4^{\circ} \mathrm{C}$ on a rotating device. The beads were separated from the solution using a magnetic stand and $2 \%$ was taken out from the cleared solution as $2 \%$ input sample. The antibody of interest was added to the remaining cleared solution and incubated overnight at $4^{\circ} \mathrm{C}$. For immunoprecipitation, beads were added to the samples and incubated at $4^{\circ} \mathrm{C}$ for $6 \mathrm{~h}$, followed by washing two times each with low salt buffer, high salt buffer, $\mathrm{LiCl}$ buffer, and Tris-HCl EDTA buffer. Samples were incubated at $65^{\circ} \mathrm{C}$ for $1 \mathrm{~h}$ to elute the DNA. Both the ChIP and input samples were incubated overnight at $65^{\circ} \mathrm{C}$ for reverse crosslinking. DNA was purified with a QIAquick PCR purification kit (Qiagen). For quantitative PCR, primers spanning the del-GUS junction yielding a 500-bp amplicon was used. EIF4 and TA3 served as the negative controls. The percent input method was used to normalize the resulting qPCR data. Primers used for qPCR are listed in Supplementary Table 3.

\section{Confocal microscopy}

To observe GFP signals in FMs, vibratome sections were produced using the primary floral bud clusters of the main inflorescence shoot. After removal of young floral buds up to stage 8 by forceps, tissues were embedded in 5\% (w/v) agar. The resulting agar blocks were sliced with a DTK$1000 \mathrm{~N}$ vibratome (Dosaka). Sections were placed on glass slides (Matsunami), mounted with a drop of water, covered by a cover glass (Matsunami), and observed with a TCS SP8 confocal microscope (Leica). At least six FMs from different floral developmental stages and temperature conditions were observed. Representative images are shown in the figures.

\section{Acknowledgements:}

We thank Akie Takahashi and Hiroko Egashira for technical assistance and Sachi Ando for checking the draft of this manuscript. Computations were partially performed on the National Institute of Genetics (NIG) supercomputer at the Research Organization of Information and Systems, NIG. This work was supported by a grant from a JSPS KAKENHI Grant-in-Aid for Scientific Research B (18H02465), Grant-in-Aid for Transformative Research Areas (A) (No. 21H05663), a grant from the SECOM Science and Technology Foundation to N.Y., a grant from the Ohsumi Frontier Science Foundation to N.Y., a JSPS KAKENHI Grant-in-Aid for Scientific Research on Innovative Areas (20H04888), a JSPS KAKENHI Grant-in-Aid for Scientific Research A (20H00470), and a Grantin-Aid for challenging Exploratory Research (No. 21K19266) to T.I. 


\section{Author contributions:}

Conceptualization: N.Y. (lead) and all other authors (supporting); data curation: N.Y.; formal analysis: M.A.P., H.S., K.M., and N.Y.; funding acquisition: N.Y. and T.I.; investigation: M.A.P., H.S., K.M., and N.Y (lead) and Z. H., L.S.L. and N.K. (supporting); project administration: N.Y.; software: N.Y. (lead) and M.A.P.; supervision: N.Y. and T.I.; validation: N.Y.; visualization: N.Y.; writing: M.A.P. and N.Y. (original draft) and all authors (review and editing).

\section{Competing interests:}

The authors declare no competing interests.

\section{References}

1. Sablowski, R. Flowering and determinacy in Arabidopsis. J Exp Bot 58, 899-907 (2007).

2. Liu, X. et al. AGAMOUS Terminates Floral Stem Cell Maintenance in Arabidopsis by Directly Repressing WUSCHEL through Recruitment of Polycomb Group Proteins. The Plant Cell 23, 3654-3670 (2011).

3. Shang, E., Ito, T. \& Sun, B. Control of floral stem cell activity in Arabidopsis. Plant Signal Behav 14, (2019).

4. Xu, Y., Yamaguchi, N., Gan, E.-S. \& Ito, T. When to stop: an update on molecular mechanisms of floral meristem termination. $J$ Exp Bot 70, 1711-1718 (2019).

5. Sun, B. \& Ito, T. Regulation of floral stem cell termination in Arabidopsis. Front Plant Sci $\mathbf{6}$, (2015).

6. Yanofsky, M. F. et al. The protein encoded by the Arabidopsis homeotic gene agamous resembles transcription factors. Nature 346, 35-39 (1990).

7. Mayer, K. F. X. et al. Role of WUSCHEL in Regulating Stem Cell Fate in the Arabidopsis Shoot 
Meristem. Cell 95, 805-815 (1998).

8. Lohmann, J. U. et al. A Molecular Link between Stem Cell Regulation and Floral Patterning in Arabidopsis. Cell 105, 793-803 (2001).

9. Lenhard, M., Bohnert, A., Jürgens, G. \& Laux, T. Termination of Stem Cell Maintenance in Arabidopsis Floral Meristems by Interactions between WUSCHEL and AGAMOUS. Cell 105, 805-814 (2001).

10. Pelayo, M. A., Yamaguchi, N. \& Ito, T. One factor, many systems: the floral homeotic protein AGAMOUS and its epigenetic regulatory mechanisms. Current Opinion in Plant Biology 61, 102009 (2021).

11. Yamaguchi, N., Huang, J., Xu, Y., Tanoi, K. \& Ito, T. Fine-tuning of auxin homeostasis governs the transition from floral stem cell maintenance to gynoecium formation. Nat Commun 8, 1125 (2017).

12. Yamaguchi, N. et al. Chromatin-mediated feed-forward auxin biosynthesis in floral meristem determinacy. Nat Commun 9, 5290 (2018).

13. Payne, T., Johnson, S. D. \& Koltunow, A. M. KNUCKLES (KNU) encodes a C2H2 zinc-finger protein that regulates development of basal pattern elements of the Arabidopsis gynoecium. Development 131, 3737-3749 (2004).

14. Sun, B., Xu, Y., Ng, K.-H. \& Ito, T. A timing mechanism for stem cell maintenance and 
differentiation in the Arabidopsis floral meristem. Genes Dev 23, 1791-1804 (2009).

15. Sun, B. et al. Timing Mechanism Dependent on Cell Division Is Invoked by Polycomb Eviction in Plant Stem Cells. Science 343, (2014).

16. Bollier, N. et al. At-MINI ZINC FINGER2 and Sl-INHIBITOR OF MERISTEM ACTIVITY, a Conserved Missing Link in the Regulation of Floral Meristem Termination in Arabidopsis and Tomato. The Plant Cell 30, 83-100 (2018).

17. Sun, B. et al. Integration of Transcriptional Repression and Polycomb-Mediated Silencing of WUSCHEL in Floral Meristems. The Plant Cell 31, 1488-1505 (2019).

18. Shang, E. et al. Robust control of floral meristem determinacy by position-specific multifunctions of KNUCKLES. PNAS 118, (2021).

19. Kwaśniewska, K. et al. Expression of KNUCKLES in the Stem Cell Domain Is Required for Its Function in the Control of Floral Meristem Activity in Arabidopsis. Frontiers in Plant Science $\mathbf{1 2}$ $1479(2021)$.

20. Huang, R., Huang, T. \& Irish, V. F. Do Epigenetic Timers Control Petal Development? Front Plant Sci 12, 709360 (2021).

21. Ikeuchi, M., Iwase, A. \& Sugimoto, K. Control of plant cell differentiation by histone modification and DNA methylation. Current Opinion in Plant Biology 28, 60-67 (2015).

22. Yamaguchi, N. The epigenetic mechanisms regulating floral hub genes and their potential for 
manipulation. Journal of Experimental Botany erab490 (2021) doi:10.1093/jxb/erab490.

23. Ó’Maoiléidigh, D. S. et al. Control of Reproductive Floral Organ Identity Specification in Arabidopsis by the C Function Regulator AGAMOUS. The Plant Cell 25, 2482-2503 (2013).

24. Sohlberg, J. J. et al. STY1 regulates auxin homeostasis and affects apical-basal patterning of the Arabidopsis gynoecium. The Plant Journal 47, 112-123 (2006).

25. Aoyanagi, T., Ikeya, S., Kobayashi, A. \& Kozaki, A. Gene Regulation via the Combination of Transcription Factors in the INDETERMINATE DOMAIN and GRAS Families. Genes 11, 613 (2020).

26. Širl, M. et al. At-Hook Motif Nuclear Localised Protein 18 as a Novel Modulator of Root System Architecture. International Journal of Molecular Sciences 21, 1886 (2020).

27. Nagano, Y., Furuhashi, H., Inaba, T. \& Sasaki, Y. A novel class of plant-specific zinc-dependent DNA-binding protein that binds to A/T-rich DNA sequences. Nucleic Acids Research 29, 4097 $4105(2001)$.

28. Xiao, J. et al. Cis and trans determinants of epigenetic silencing by Polycomb repressive complex 2 in Arabidopsis. Nature Genetics 49, 1546-1552 (2017).

29. Yoon, K.-J., Vissers, C., Ming, G. \& Song, H. Epigenetics and epitranscriptomics in temporal patterning of cortical neural progenitor competence. J Cell Biol 217, 1901-1914 (2018).

30. Eccleston, A., Cesari, F. \& Skipper, M. Transcription and epigenetics. Nature 502, 461-461 
(2013).

31. Mozgova, I., Köhler, C. \& Hennig, L. Keeping the gate closed: functions of the polycomb repressive complex PRC2 in development. The Plant Journal 83, 121-132 (2015).

32. Bemer, M. \& Grossniklaus, U. Dynamic regulation of Polycomb group activity during plant development. Current Opinion in Plant Biology 15, 523-529 (2012).

33. Mozgova, I. \& Hennig, L. The Polycomb Group Protein Regulatory Network. Annu. Rev. Plant Biol. 66, 269-296 (2015).

34. Kim, D.-H. \& Sung, S. Polycomb-Mediated Gene Silencing in Arabidopsis thaliana. Mol Cells 37, 841-850 (2014).

35. Ramachandran, S. \& Henikoff, S. Replicating nucleosomes. Science Advances 1, e1500587 (2015).

36. Alabert, C. et al. Two distinct modes for propagation of histone PTMs across the cell cycle. Genes Dev. 29, 585-590 (2015).

37. Lai, W. K. M. \& Pugh, B. F. Understanding nucleosome dynamics and their links to gene expression and DNA replication. Nature Reviews Molecular Cell Biology 18, 548-562 (2017).

38. Bogliotti, Y. S. \& Ross, P. J. Mechanisms of histone H3 lysine 27 trimethylation remodeling during early mammalian development. Epigenetics 7, 976-981 (2012).

39. Zik, M. \& Irish, V. F. Flower Development: Initiation, Differentiation, and Diversification. Annu. 
Rev. Cell Dev. Biol. 19, 119-140 (2003).

40. Mambro, R. D. et al. Auxin minimum triggers the developmental switch from cell division to cell differentiation in the Arabidopsis root. PNAS 114, E7641-E7649 (2017).

41. Harashima, H. \& Sekine, M. Cyclin-Dependent Protein Kinases in the Control of Cell Cycle in Plants. in Protein Kinases and Stress Signaling in Plants 347-368 (John Wiley \& Sons, Ltd, 2020). doi:10.1002/9781119541578.ch14.

42. Kumar, N. \& Larkin, J. C. Why do plants need so many cyclin-dependent kinase inhibitors? Plant Signaling \& Behavior 12, e1282021 (2017).

43. Veylder, L. D. et al. Functional Analysis of Cyclin-Dependent Kinase Inhibitors of Arabidopsis. The Plant Cell 13, 1653-1668 (2001).

44. Churchman, M. L. et al. SIAMESE, a Plant-Specific Cell Cycle Regulator, Controls Endoreplication Onset in Arabidopsis thaliana. Plant Cell 18, 3145-3157 (2006).

45. Ito, T. et al. The homeotic protein AGAMOUS controls microsporogenesis by regulation of SPOROCYTELESS. Nature 430, 356-360 (2004).

46. Gómez-Mena, C., Folter, S. de, Costa, M. M. R., Angenent, G. C. \& Sablowski, R. Transcriptional program controlled by the floral homeotic gene AGAMOUS during early organogenesis. Development 132, 429-438 (2005).

47. Barton, M. K. \& Poethig, R. S. Formation of the shoot apical meristem in Arabidopsis thaliana: 
an analysis of development in the wild type and in the shoot meristemless mutant. Development 119, 823-831 (1993).

48. Han, G. et al. Arabidopsis ZINC FINGER PROTEIN1 Acts Downstream of GL2 to Repress Root Hair Initiation and Elongation by Directly Suppressing bHLH Genes. The Plant Cell 32, 206-225 (2020).

49. Bosco, C. D. et al. Inactivation of the Chloroplast ATP Synthase $\gamma$ Subunit Results in High Nonphotochemical Fluorescence Quenching and Altered Nuclear Gene Expression in Arabidopsis thaliana. J. Biol. Chem. 279, 1060-1069 (2004).

50. Hanada, K. et al. Functional Compensation of Primary and Secondary Metabolites by Duplicate Genes in Arabidopsis thaliana. Mol Biol Evol 28, 377-382 (2011).

51. Ma, C., Haslbeck, M., Babujee, L., Jahn, O. \& Reumann, S. Identification and Characterization of a Stress-Inducible and a Constitutive Small Heat-Shock Protein Targeted to the Matrix of Plant Peroxisomes. Plant Physiology 141, 47-60 (2006).

52. Riechmann, J. L. et al. Arabidopsis Transcription Factors: Genome-Wide Comparative Analysis Among Eukaryotes. Science 290, 2105-2110 (2000).

53. Liu, S. et al. PLATZ2 negatively regulates salt tolerance in Arabidopsis seedlings by directly suppressing the expression of the CBL4/SOS3 and CBL10/SCaBP8 genes. $J$ Exp Bot 71, 5589$5602(2020)$ 
54. Gong, W. et al. Genome-Wide ORFeome Cloning and Analysis of Arabidopsis Transcription Factor Genes. Plant Physiology 135, 773-782 (2004).

55. Tian, T. et al. agriGO v2.0: a GO analysis toolkit for the agricultural community, 2017 update. Nucleic Acids Res 45, W122-W129 (2017).

56. Supek, F., Bošnjak, M., Škunca, N. \& Šmuc, T. REVIGO Summarizes and Visualizes Long Lists of Gene Ontology Terms. PLOS ONE 6, e21800 (2011).

57. Probst, A. V., Dunleavy, E. \& Almouzni, G. Epigenetic inheritance during the cell cycle. Nat Rev Mol Cell Biol 10, 192-206 (2009).

58. Polycomb gene expression and histone $\mathrm{H} 3$ lysine 27 trimethylation changes during bovine preimplantation development in: Reproduction Volume 136 Issue 6 (2008). https://rep.bioscientifica.com/view/journals/rep/136/6/777.xml.

59. Katz, A., Oliva, M., Mosquna, A., Hakim, O. \& Ohad, N. FIE and CURLY LEAF polycomb proteins interact in the regulation of homeobox gene expression during sporophyte development. The Plant Journal 37, 707-719 (2004).

60. Doyle, M. R. \& Amasino, R. M. A Single Amino Acid Change in the Enhancer of Zeste Ortholog CURLY LEAF Results in Vernalization-Independent, Rapid Flowering in Arabidopsis. Plant Physiology 151, 1688-1697 (2009).

61. Liu, J. et al. CURLY LEAF Regulates Gene Sets Coordinating Seed Size and Lipid Biosynthesis. 
Plant Physiology 171, 424-436 (2016).

62. Wigge, P. A. Ambient temperature signalling in plants. Current Opinion in Plant Biology 16, 661$666(2013)$

63. Michael, T. P. et al. Network Discovery Pipeline Elucidates Conserved Time-of-Day-Specific cisRegulatory Modules. PLOS Genetics 4, e14 (2008).

64. Cheng, Y. et al. Downregulation of multiple CDK inhibitor ICK/KRP genes upregulates the E2F pathway and increases cell proliferation, and organ and seed sizes in Arabidopsis. The Plant Journal 75, 642-655 (2013).

65. Zhou, Y., Wang, H., Gilmer, S., Whitwill, S. \& Fowke, L. C. Effects of co-expressing the plant CDK inhibitor ICK1 and D-type cyclin genes on plant growth, cell size and ploidy in Arabidopsis thaliana. Planta 216, 604-613 (2003).

66. Laurenzio, L. D. et al. The SCARECROW Gene Regulates an Asymmetric Cell Division That Is Essential for Generating the Radial Organization of the Arabidopsis Root. Cell 86, 423-433 (1996).

67. Lucas, M. et al. SHORT-ROOT Regulates Primary, Lateral, and Adventitious Root Development in Arabidopsis. Plant Physiology 155, 384-398 (2011).

68. Wong, M. M. et al. Phosphoproteomics of Arabidopsis Highly ABA-Induced1 identifies ATHook-Like10 phosphorylation required for stress growth regulation. PNAS 116, 2354-2363 
(2019).

69. Ng, K.-H., Yu, H. \& Ito, T. AGAMOUS Controls GIANT KILLER, a Multifunctional Chromatin Modifier in Reproductive Organ Patterning and Differentiation. PLOS Biology 7, e1000251 (2009).

70. Lim, P. O. et al. Overexpression of a chromatin architecture-controlling AT-hook protein extends leaf longevity and increases the post-harvest storage life of plants. The Plant Journal 52, 11401153 (2007).

71. Yadeta, K. A. et al. The Arabidopsis thaliana DNA-Binding Protein AHL19 Mediates Verticillium Wilt Resistance. MPMI 24, 1582-1591 (2011).

72. Matsushita, A., Furumoto, T., Ishida, S. \& Takahashi, Y. AGF1, an AT-Hook Protein, Is Necessary for the Negative Feedback of AtGA3ox1 Encoding GA 3-Oxidase. Plant Physiol 143, 1152-1162 (2007).

73. Lee, K. \& Seo, P. J. Coordination of matrix attachment and ATP-dependent chromatin remodeling regulate auxin biosynthesis and Arabidopsis hypocotyl elongation. PLOS ONE 12, e0181804 (2017).

74. Street, I. H., Shah, P. K., Smith, A. M., Avery, N. \& Neff, M. M. The AT-hook-containing proteins SOB3/AHL29 and ESC/AHL27 are negative modulators of hypocotyl growth in Arabidopsis. The Plant Journal 54, 1-14 (2008). 
75. Zhou, J., Wang, X., Lee, J.-Y. \& Lee, J.-Y. Cell-to-Cell Movement of Two Interacting AT-Hook Factors in Arabidopsis Root Vascular Tissue Patterning. The Plant Cell 25, 187-201 (2013).

76. Wellmer, F., Alves-Ferreira, M., Dubois, A., Riechmann, J. L. \& Meyerowitz, E. M. GenomeWide Analysis of Gene Expression during Early Arabidopsis Flower Development. PLOS Genetics 2, e117 (2006).

77. Iwata, E. et al. GIGAS CELL1, a Novel Negative Regulator of the Anaphase-Promoting Complex/Cyclosome, Is Required for Proper Mitotic Progression and Cell Fate Determination in Arabidopsis[W]. Plant Cell 23, 4382-4393 (2011).

78. Thorvaldsdóttir, H., Robinson, J. T. \& Mesirov, J. P. Integrative Genomics Viewer (IGV): highperformance genomics data visualization and exploration. Brief Bioinform 14, 178-192 (2013).

79. Chen, L., Wang, F., Wang, X. \& Liu, Y.-G. Robust one-Tube $\Omega$-PCR Strategy Accelerates Precise Sequence Modification of Plasmids for Functional Genomics. Plant Cell Physiol 54, 634-642 (2013).

80. Weigel, D. \& Glazebrook, J. Arabidopsis: A Laboratory Manual. (CSHL Press, 2002).

81. Yamaguchi, N. et al. PROTOCOLS: Chromatin Immunoprecipitation from Arabidopsis Tissues. Arabidopsis Book 12, (2014). 
a

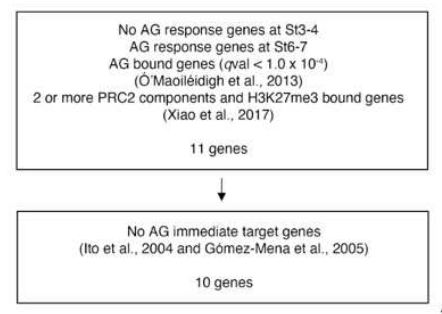

b

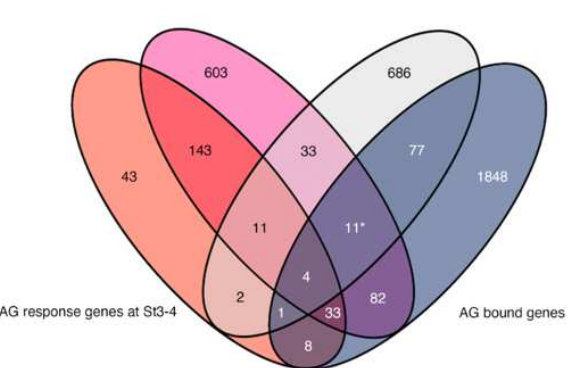

c
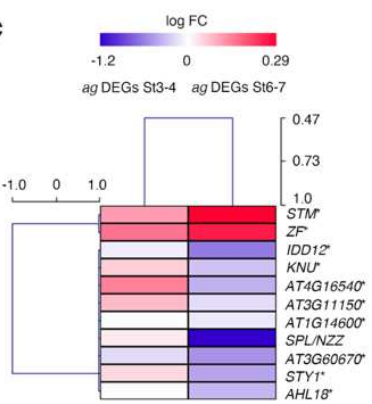

。

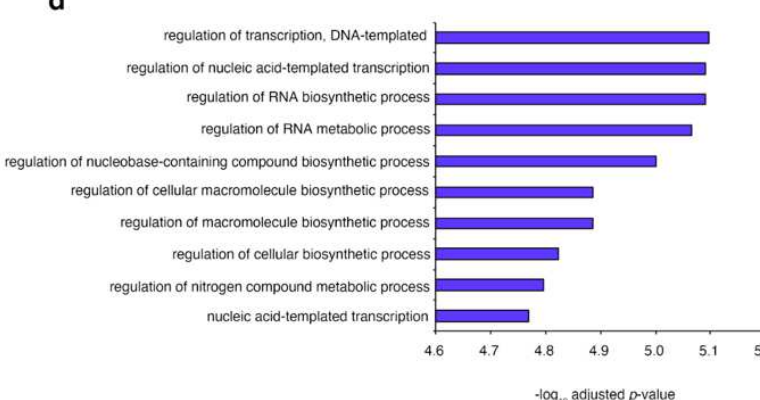

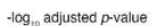

f
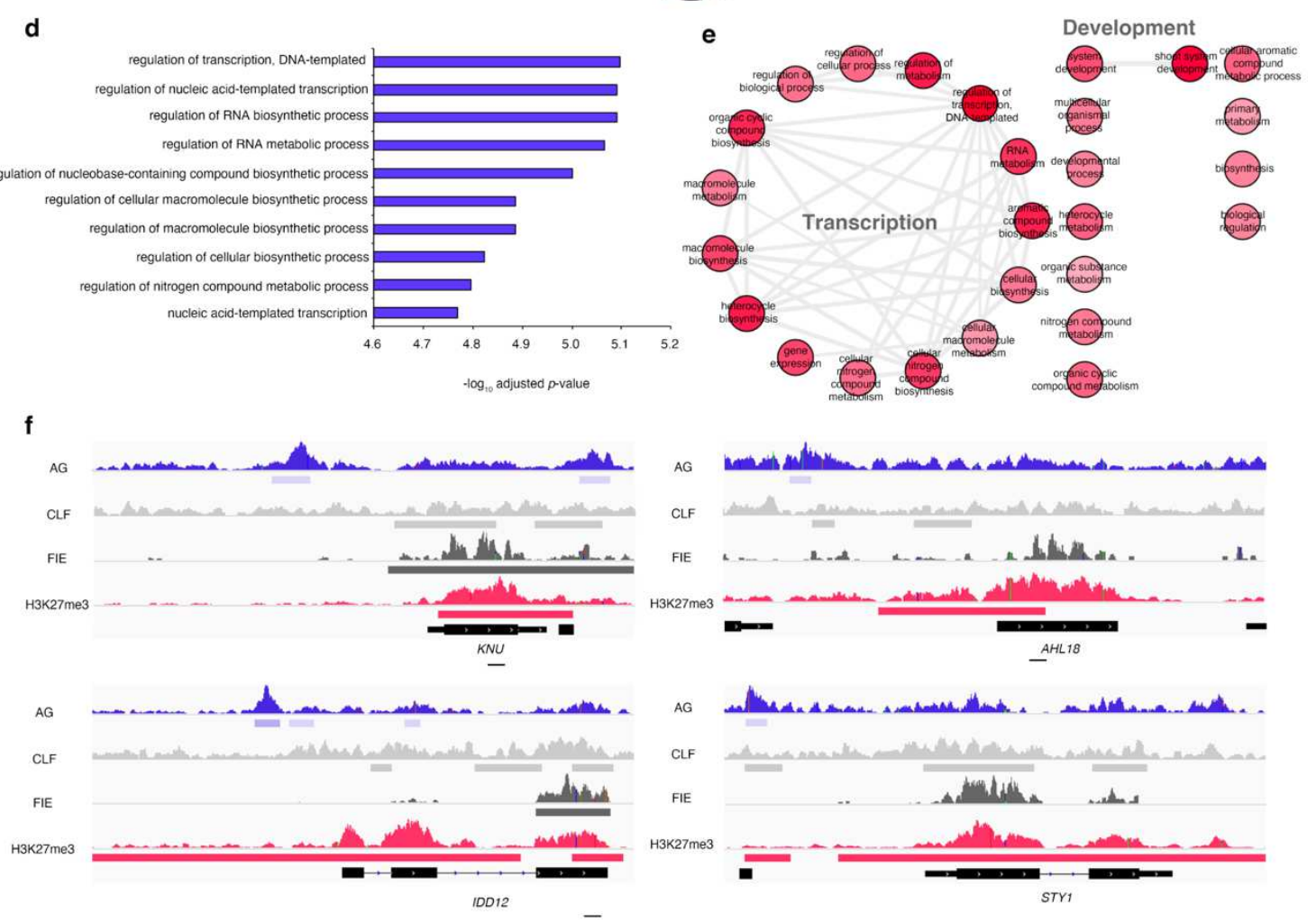

Fig. 1. Genome-wide identification of biotimer-regulated genes

a, Schematic representation of reference datasets used and selection criteria for filtering biotimerregulated genes of interest. b, Venn diagram showing the extent of overlap between gene clusters generated following the selection criteria. The 11 identified biotimer-regulated genes are indicated by an asterisk. These are AG-response genes with significant transcriptional changes at stages 6-7, are bound directly by AG and PRC2 components, and are marked by H3K27me3. c, Transcriptional heatmap of the 11 identified biotimer-regulated genes. Significant transcriptional changes occur for all genes at stages 6-7 (right) compared to stages 3-4 (left). Two genes are induced at stages 6-7 (STM and $Z F$ ) while the others are repressed (horizontal clustering, $\log _{2}$ [fold change] from -1.0 to 1.0). $\mathbf{d}$, Top ten enriched GO terms for the identified biotimer-regulated genes (excluding SPL and NZZ) generated using agriGO v2.0. Functional enrichment ranking is based on the $p$ values of significant GO terms. e, Graph-based visualization of refined GO terminology using REVIGO. The node radius 
indicates the specificity and the color shading corresponds to the $p$ values. Highly similar GO terms are linked by edges, with edge thickness indicating the degree of similarity ${ }^{56}$. f, Input-subtracted ChIPseq signals for AG, the PRC2 components CLF and FIE, and H3K27me3 at the KNU, AHL18, IDD12, and STY1 loci. Solid horizontal bars below the peaks indicate regions with significant binding. The gene models are shown as black bars and lines at the bottom of each panel. Lines indicate PCR amplicons for Fig. 2. 

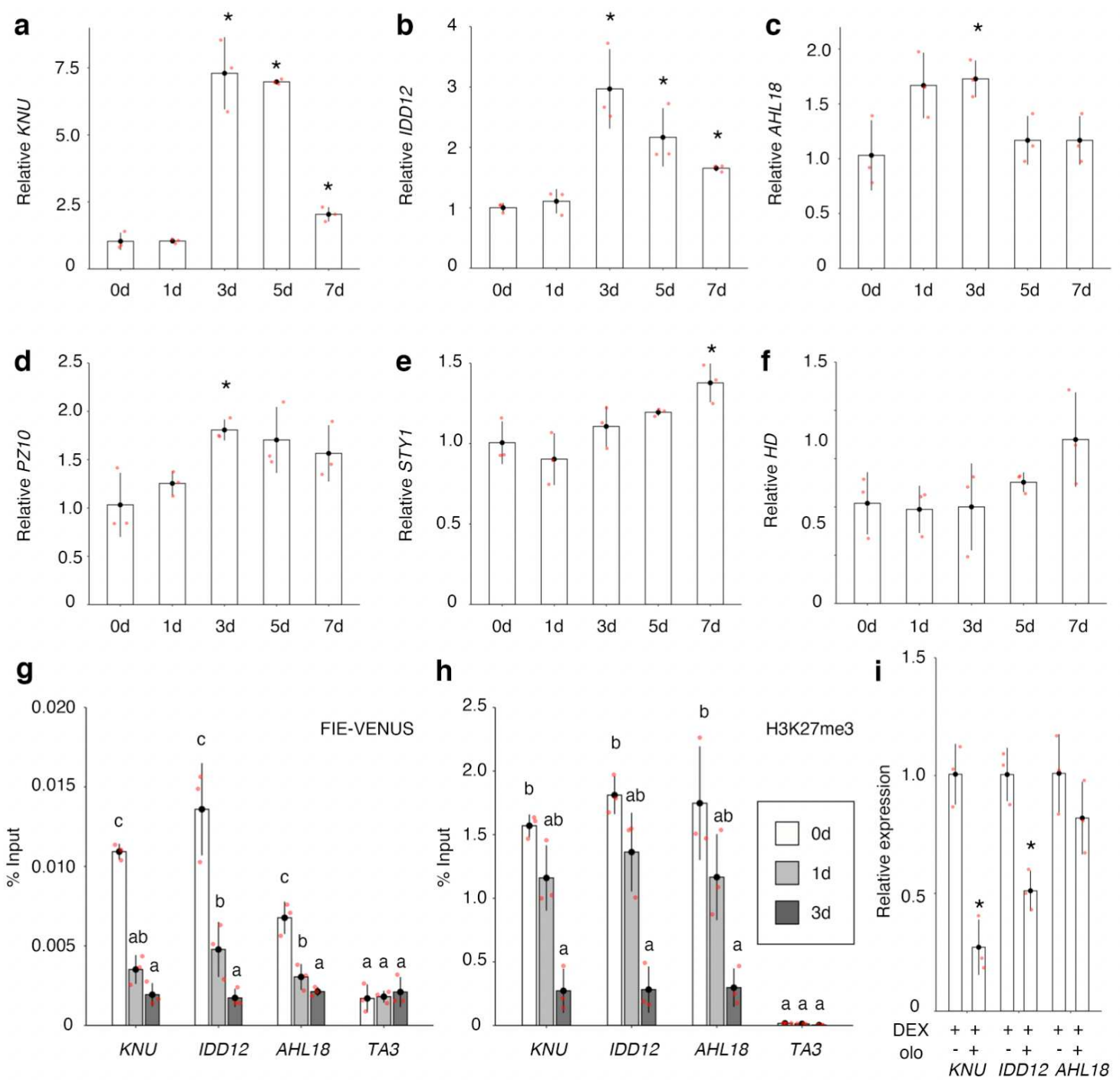

Fig. 2. Regulation of gene expression by biotimer

a-f, Relative expression levels for $K N U$ (a), IDD12 (b), AHL18 (c), PZ10 (d), STY1 (e), and HD (f) in ag-1 35S::AG-GR without and with DEX treatment, as determined by RT-qPCR. Data represent average fold change \pm standard errors of three biological replicates $(n=3)$. Significant differences were determined using a two-tailed Student's $t$-test $\left({ }^{*} p<0.05\right)$. TUBULIN 2 (TUB2) served as the reference transcript. g, FIE-VENUS enrichment in ap1 cal 35S::AP1-GR without and with DEX treatment. Data represent average of \% Input \pm standard errors from three biological replicates $n=3$ ). Significant differences were determined using a two-tailed Student's $t$-test $\left({ }^{*} p<0.05\right)$. TA3 served as the negative control. $\mathbf{h}, \mathrm{H} 3 \mathrm{~K} 27 \mathrm{me} 3$ enrichment in ap1 cal 35S::AP1-GR without and with DEX treatment. Data represent average of $\%$ Input \pm standard errors from three biological replicates $n=3$ ). Significant differences were determined using a two-tailed Student's $t$-test $\left({ }^{*} p<0.05\right)$. TA3 served as the negative control. PCR amplicons are shown in Fig. 1f. i, Relative expression levels for $K N U$, 
IDD12, and AHL18 in DEX-treated ag-1 35S::AG-GR without and with olo treatment, as determined by RT-qPCR. Data represent average fold change \pm standard errors of three biological replicates $(\mathrm{n}=$ 3). Student's $t$-test, $p<0.05$. 
a

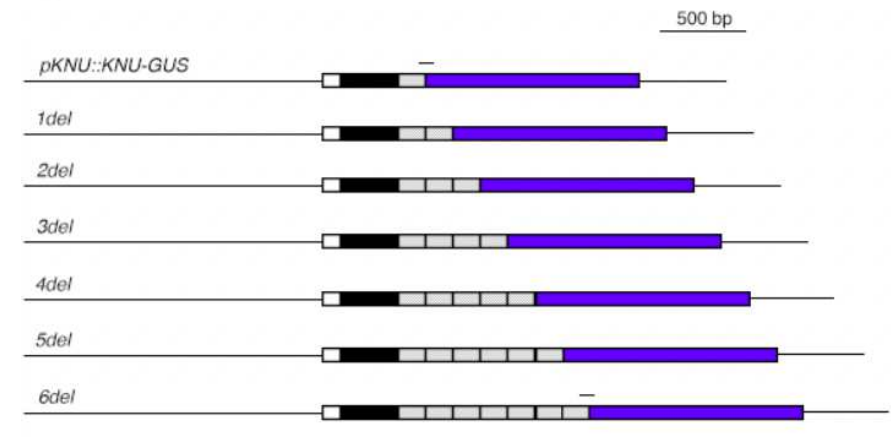

b
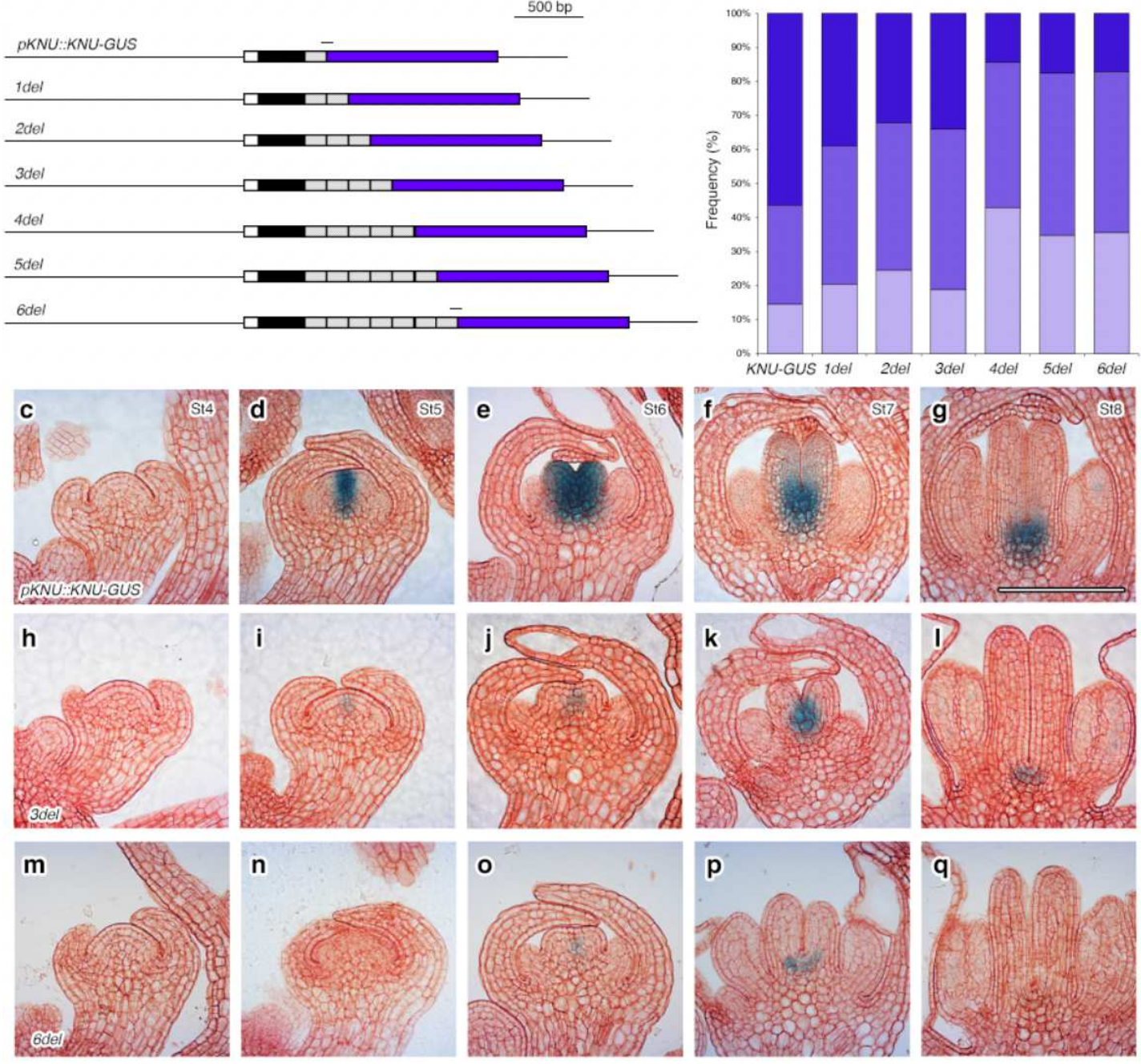

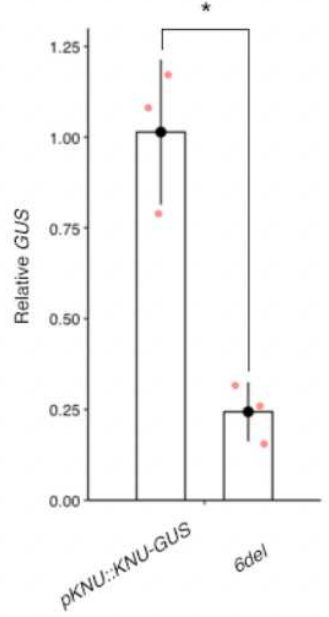

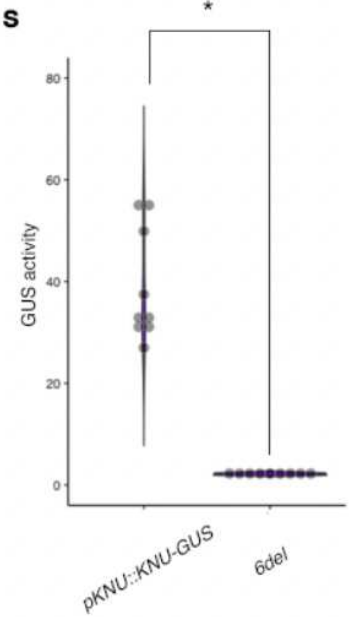

t

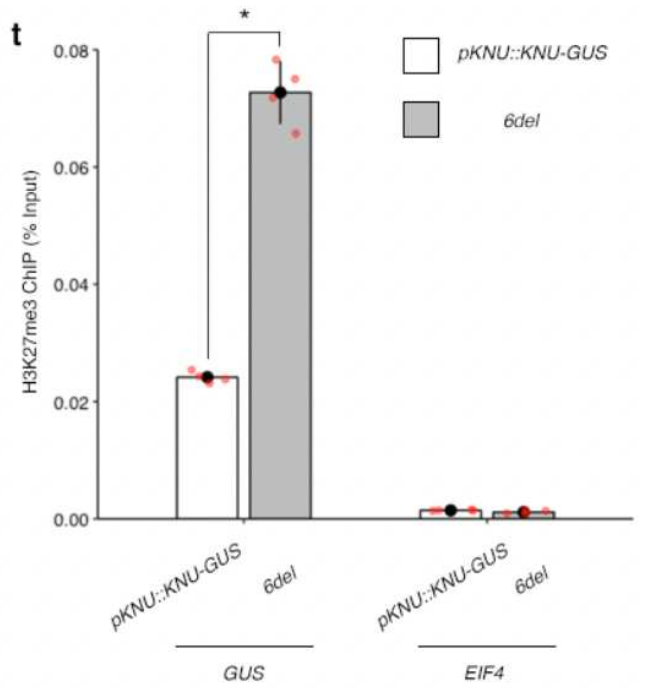

Fig. 3. Extension of the $K N U$ coding sequence prolongs the delay in $K N U$ activation and reduces $K N U$ expression 
a, Schematic diagrams of the $K N U-G U S$ series of constructs. Solid black line, $K N U$ promoter region; white bars, 5' untranslated region (5' UTR); black bars, 255 bp of $K N U$ coding sequence; gray, 231bp del sequence (one [ $1 \mathrm{del}]$ to six [6del] copies); blue bars, GUS gene. The line above the del and GUS gene junction is a PCR amplicon used for ChIP-qPCR. b, Variation in GUS activity levels in $p K N U:: K N U-G U S$ and del $\mathrm{T}_{1}$ plants. The dark blue, blue, and light blue bars represent strong, intermediate, and weak GUS signal intensity, respectively. A $X^{2}$ test was used to test for significant differences among the del lines ( $\left.\mathrm{n}>35,{ }^{*} p<0.05\right)$. $\mathbf{c}-\mathbf{q}$, Representative longitudinal GUS sections from stage 4-8 floral buds from $p K N U:: K N U-G U S(\mathrm{c}-\mathrm{g}), 3 \operatorname{del}(\mathrm{h}-1)$, and $6 \operatorname{del}(\mathrm{m}-\mathrm{q})$. Cell walls were stained with neutral red dye. Scale bar $=50 \mu \mathrm{m} . \mathbf{r}, G U S$ transcript levels in $p K N U:: K N U$-GUS and 6 del $\mathrm{T}_{1}$ plants, as determined by RT-qPCR. Data represent average fold change \pm standard errors of three biological replicates $(\mathrm{n}=3)$. Significant differences were determined using a two-tailed Student's $t$-test $\left({ }^{*} p<0.05\right) . T U B 2$ served as the reference transcript. s, GUS activity levels, as determined by MUG assay. Data represent average GUS activity $(\mathrm{nmol} / \mathrm{mg} / \mathrm{h}) \pm$ standard errors from nine biological replicates $(\mathrm{n}=9)$. Significant differences were determined using a two-tailed Student's $t$-test $\left({ }^{*} p<\right.$ 0.05). t, H3K27me3 enrichment in $p K N U:: K N U-G U S$ and 6 del $\mathrm{T}_{1}$ plants. Data represent average \% Input \pm standard errors from four biological replicates $(n=4)$. Significant differences were determined using a two-tailed Student's $t$-test $\left({ }^{*} p<0.05\right)$. EIF4A1 served as the negative control. 

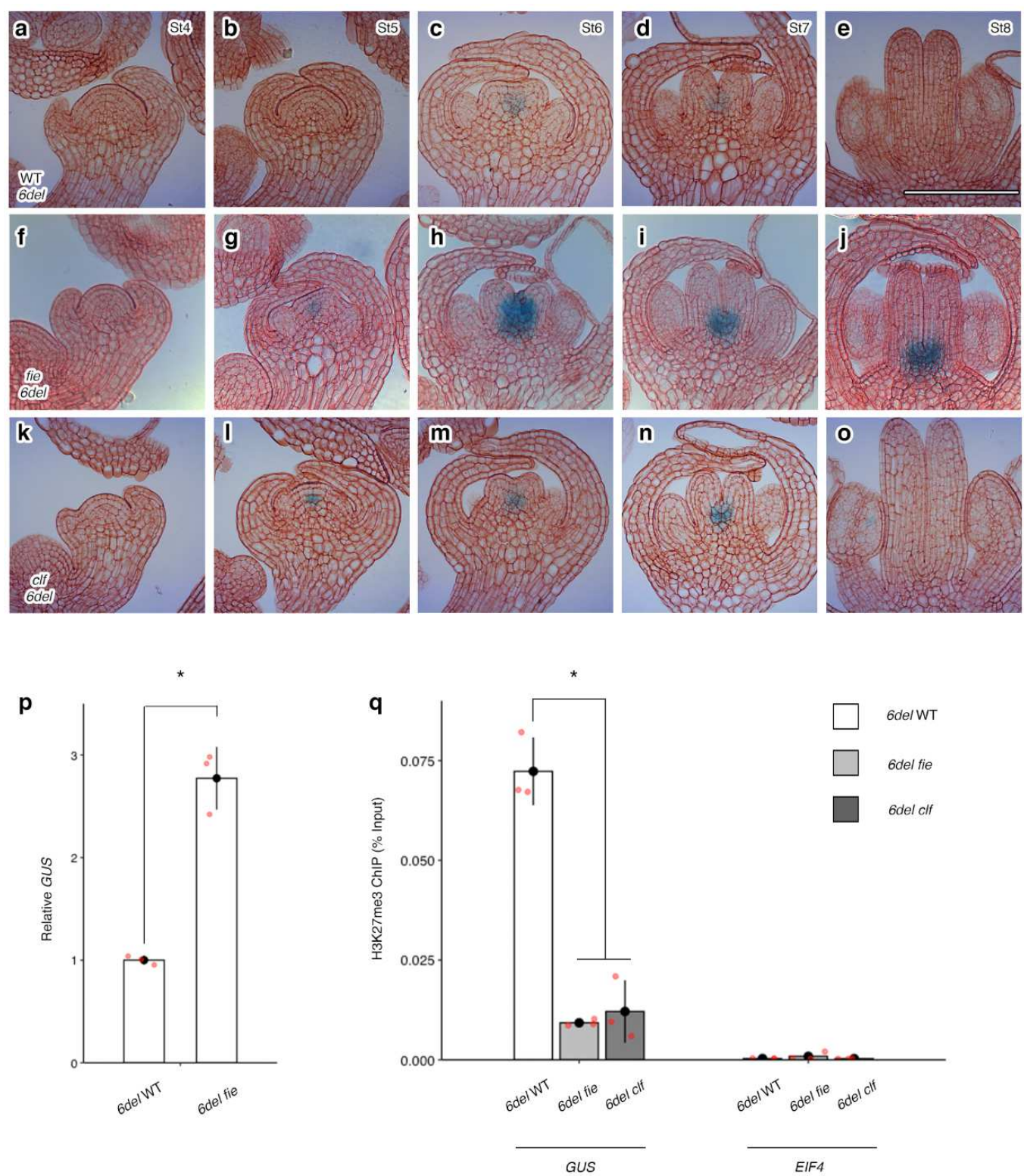

Fig. 4. PRC2 deposits H3K27me3 on the del motif

a-o, Representative longitudinal GUS sections from stage 4-8 floral buds for WT, fie, and clf transformants harboring $6 \mathrm{del}$. Cell walls were stained with neutral red dye. Scale bar $=50 \mu \mathrm{m} . \mathbf{p}, G U S$ transcript levels derived from $6 \mathrm{del}$ in WT and fie transformants, as determined by RT-qPCR. Data represent average fold change \pm standard errors of three biological replicates $(n=3)$. A two-tailed Student's $t$-test was used to determine significant differences $\left({ }^{*} p<0.05\right)$. TUB2 served as the reference transcript. q, H3K27me3 enrichment over 6del in WT compared to fie and clf. Data represent average $\%$ Input \pm standard errors from three independent experiments $(n=3)$. A two-tailed Student's $t$-test was used to determine significant differences $\left({ }^{*} p<0.05\right)$. EIF4A1 served as the negative control. 

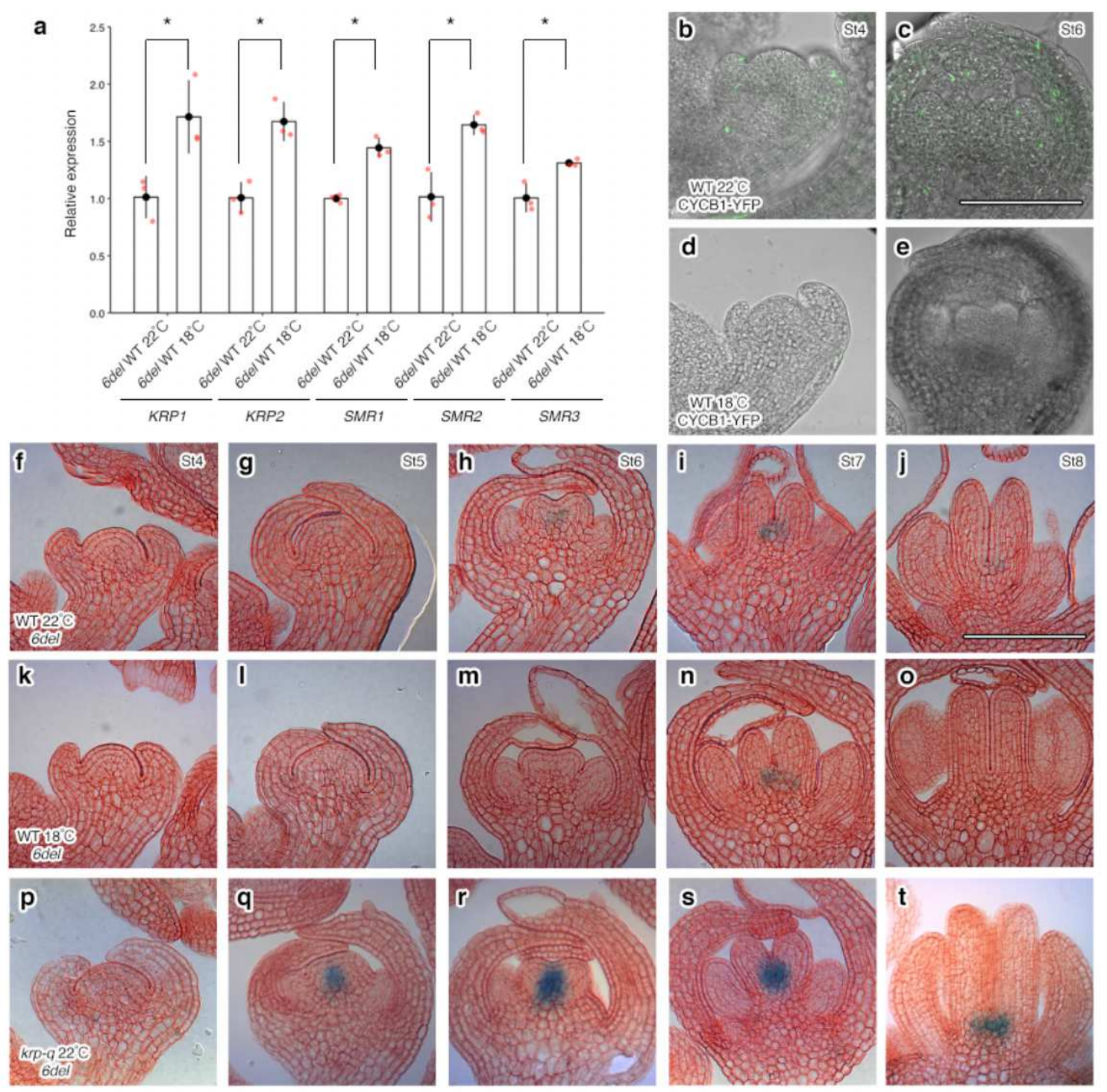

u

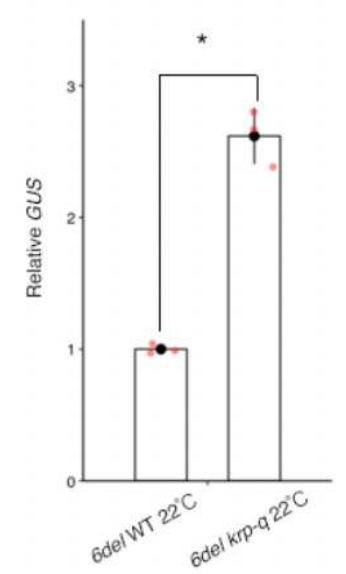

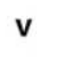

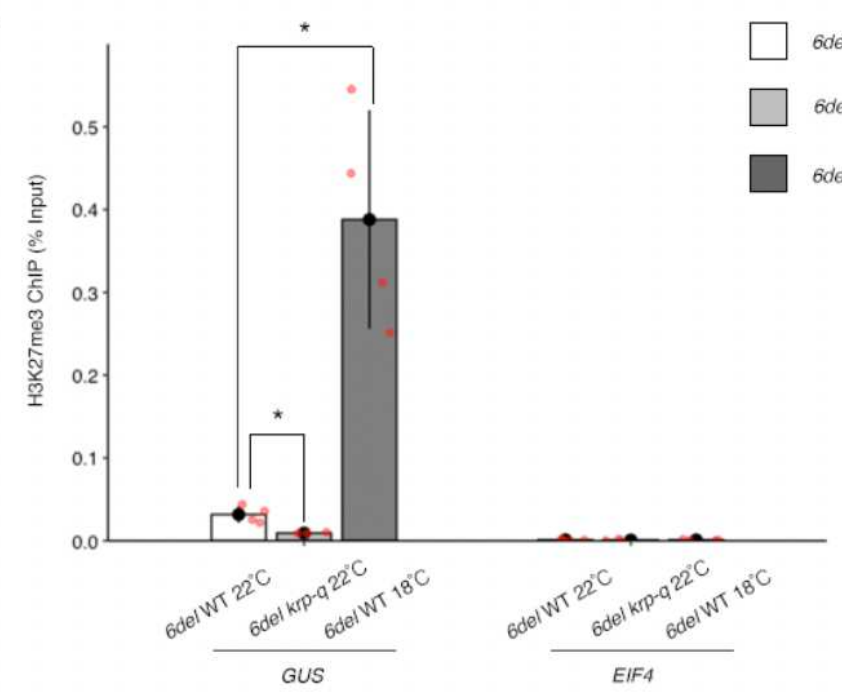




\section{Fig. 5. The dilution of $\mathrm{H} 3 \mathrm{~K} 27 \mathrm{me} 3$ at $\mathrm{del}$ is cell cycle-dependent}

a, Cell cycle-related gene expression levels derived from $6 \mathrm{del}$ in WT, as determined by RT-qPCR. Data represent average fold change \pm standard errors of three biological replicates $(n=3)$. A two-tailed Student's $t$-test was used to determine significant differences $\left({ }^{*} p<0.05\right)$. TUB2 served as the reference transcript. b-e, Representative longitudinal YFP sections from stage 4 (b, d) and stage 6 (c, e) floral buds from WT harboring the CYCB1;1-YFP construct grown at $22^{\circ} \mathrm{C}(\mathrm{b}, \mathrm{c})$, and at $18^{\circ} \mathrm{C}(\mathrm{d}, \mathrm{e}) . \mathbf{f}-\mathbf{t}$, Representative longitudinal GUS sections from stage 4-8 floral buds from WT harboring the 6 del construct $(\mathrm{f}-\mathrm{j}), 6$ del $k r p-q$ grown at $22^{\circ} \mathrm{C}(\mathrm{k}-\mathrm{o})$, and 6 del in WT grown at $18^{\circ} \mathrm{C}(\mathrm{p}-\mathrm{t})$. Cell walls were stained with neutral red dye. Scale bar $=50 \mu \mathrm{m} . \mathbf{u}, G U S$ transcript levels derived from WT and $k r p-q$ transformants harboring $6 \mathrm{del}$ and grown at $22^{\circ} \mathrm{C}$, as determined by RT-qPCR using RNA isolated from floral bud clusters containing flowers until stage 12. Data represent average fold change \pm standard errors of three biological replicates $(\mathrm{n}=3)$. A two-tailed Student's $t$-test was used to determine significant differences $\left({ }^{*} p<0.05\right)$. TUB2 served as the reference transcript. v, H3K27me3 enrichment at $6 \mathrm{del}$ in WT and $k r p-q$ transformants harboring $6 \mathrm{del}$ grown at $22^{\circ} \mathrm{C}$ or $18^{\circ} \mathrm{C}$. Samples used were floral bud clusters containing flowers until stage 12. Data represent average $\%$ Input \pm standard errors from three independent experiments $(\mathrm{n}=3)$. A two-tailed Student's $t$-test was used to determine significant differences $\left({ }^{*} p<0.05\right)$. EIF $4 A 1$ served as the negative control. 


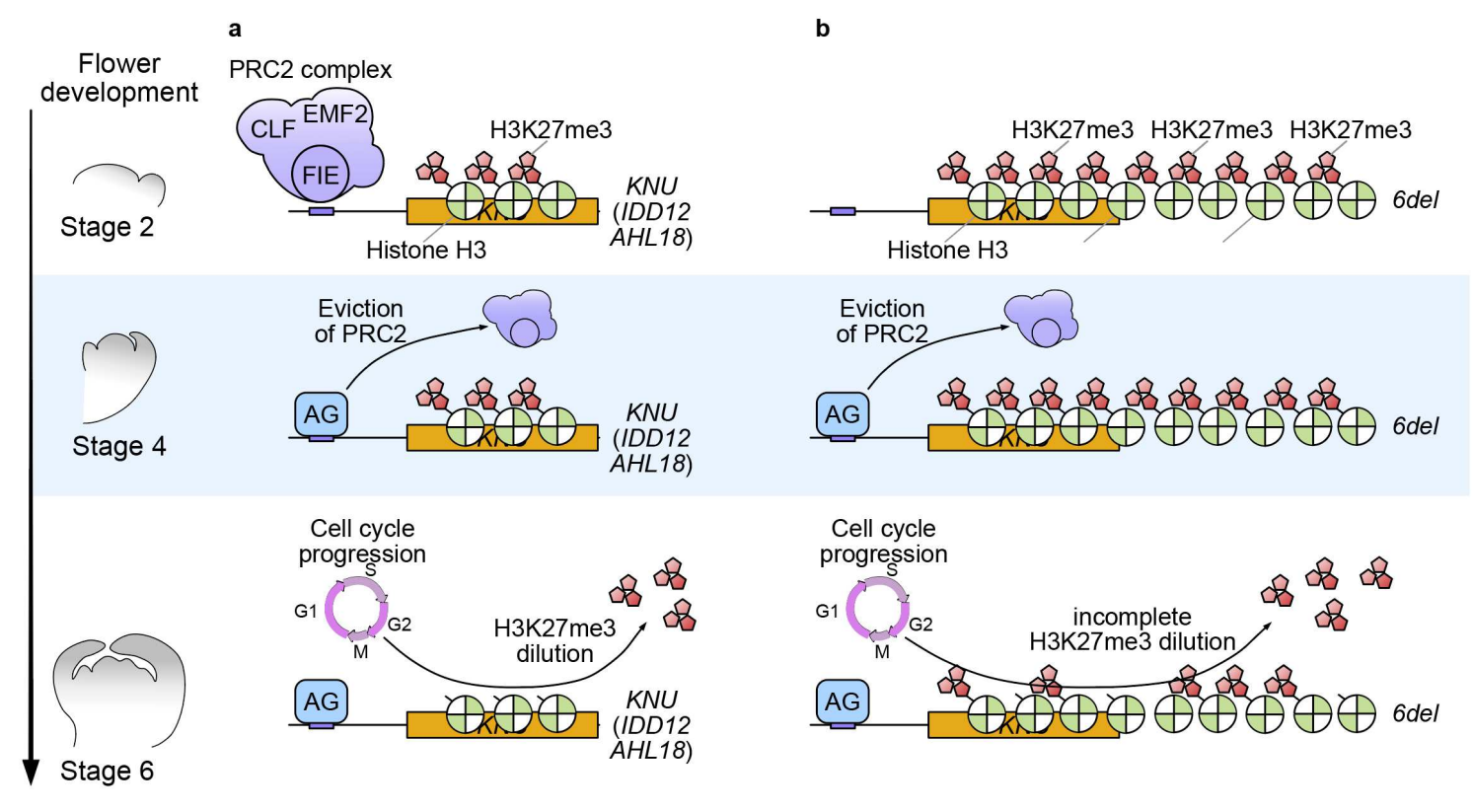

Fig. 6. Proposed model for biotimer-regulated genes and their manipulation

a, Biotimer regulation for $K N U, I D D 12$, and AHL18 loci. Above: Prior to AG accumulation, PRC2 deposits H3K27me3 along the gene bodies of $K N U$, IDD12, and AHL18 loci for transcriptional silencing. Middle: At stage 3 of flower development, AG accumulation leads to PRC2 displacement at the $K N U, I D D 12$, and $A H L 18$ loci. Below: By stage 6, most proliferating cells are free of H3K27me3 marks at the $K N U, I D D 12$, and $A H L 18$ loci and biotimer-regulated genes are transcribed. b, Biotimer manipulation by iterative addition of $\mathrm{H} 3 \mathrm{~K} 27 \mathrm{me} 3$-enriched regions. The first two steps are identical to the endogenous regulation of $K N U, I D D 12$, and AHL18 expression. Below: Even at stage 6, $\mathrm{H} 3 \mathrm{~K} 27 \mathrm{me} 3$ dilution in most cells remains incomplete and hence 6 del expression is delayed. 
Supplementary materials for

A histone modification-based synthetic circuit to engineer temporal gene expression in Arabidopsis floral meristems

Margaret Anne Pelayo ${ }^{1}$, Haruka Sawada ${ }^{1}$, Kasumi Matsushita ${ }^{1}$, Liang Sheng Looi ${ }^{1}$, Naoya Katagiri ${ }^{1}$, Nobutoshi Yamaguchi ${ }^{1 *}$ and Toshiro Ito ${ }^{1 *}$

*Correspondence to: nobuy@bs.naist.jp; itot@bs.naist.jp 
a

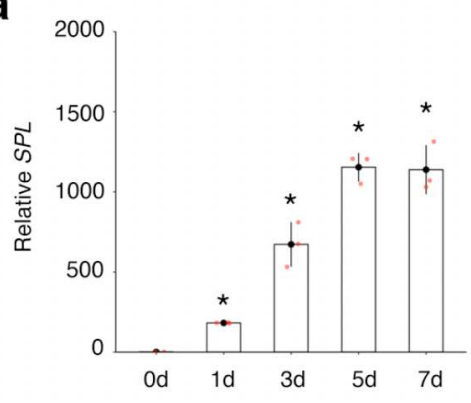

b

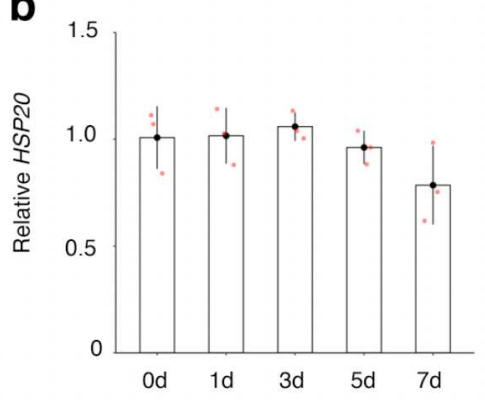

Extended Data Fig. 1. SPL/NZZ is immediately induced in DEX-treated ag-1 35S:AG-GR plants a, b, Relative expression levels for $S P L$ (a), and $H S P 20$ (b) in ag-1 $35 S:: A G-G R$ without and with DEX treatment, as determined by RT-qPCR. Data represent average fold change \pm standard errors of three biological replicates $(\mathrm{n}=3)$. Significant differences were determined using a two-tailed Student's $t$-test $\left({ }^{*} p<0.05\right)$. TUBULIN $2($ TUB2) served as the reference transcript. 


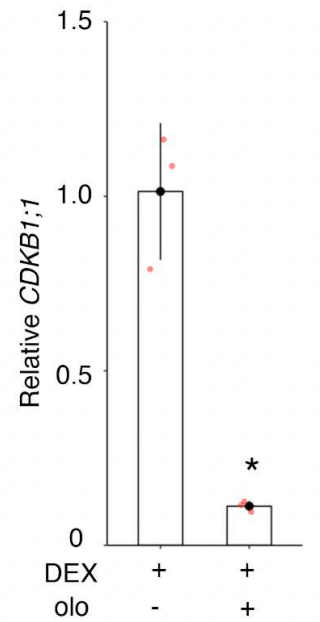

Extended Data Fig. 2. CDKB1;1 is repressed by olo in DEX-treated ag-1 35S:AG-GR plants

Relative expression levels for $C D K B 1 ; 1$ in DEX-treated ag-1 $35 S:: A G-G R$ without and with olo treatment, as determined by RT-qPCR. Data represent average fold change \pm standard errors of three biological replicates $(\mathrm{n}=3)$. Student's $t$-test, $p<0.05$. 


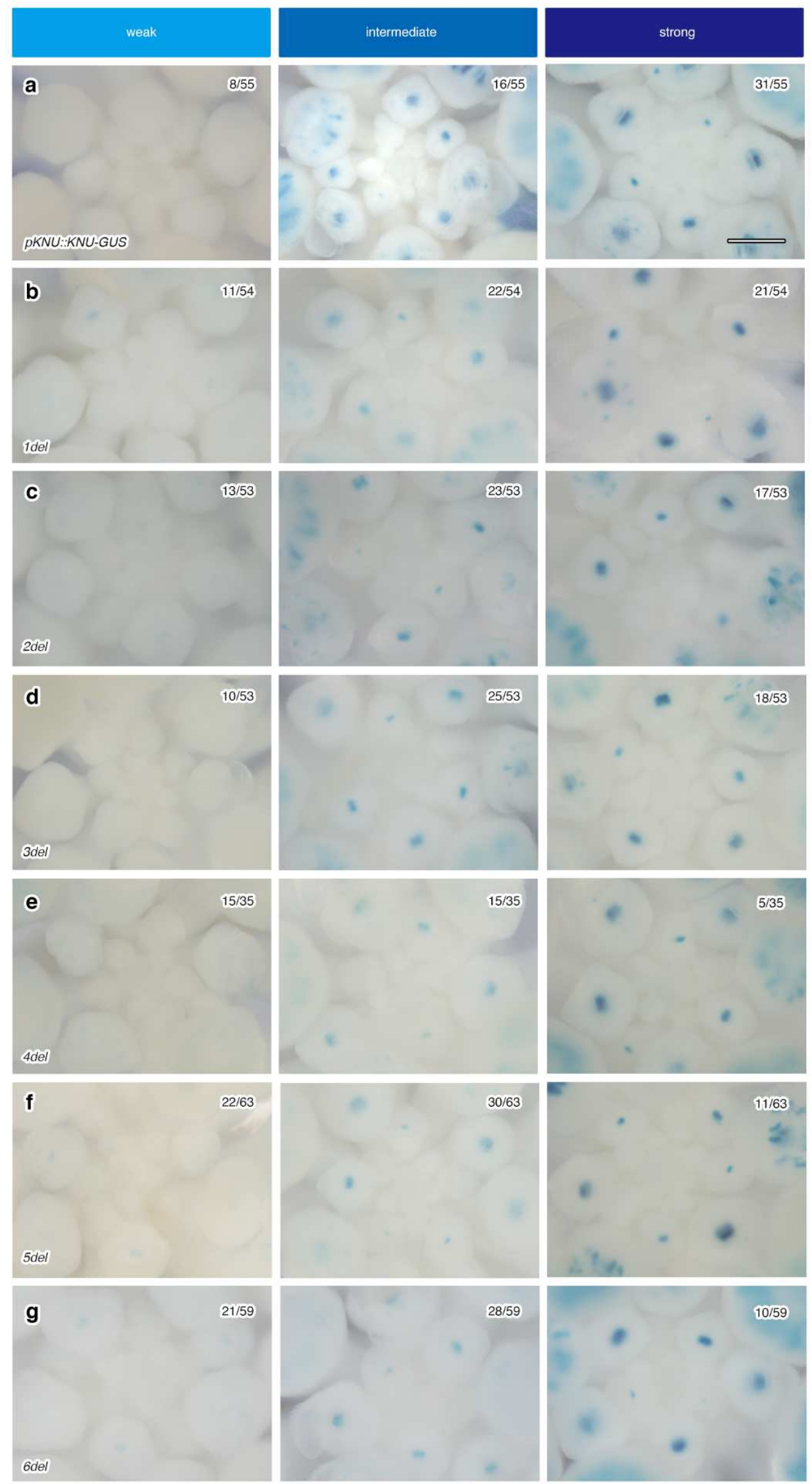


Extended Data Fig. 3. GUS staining pattern in $T_{1}$ plants can be categorized into three different categories

a-g, GUS signal was observed in floral stages 6-8 and categorized as weak, intermediate or strong in WT (a), $1 \operatorname{del}(\mathrm{b}), 2 \operatorname{del}(\mathrm{c}), 3 \operatorname{del}(\mathrm{d}), 4 \operatorname{del}(\mathrm{e}), 5 \operatorname{del}(\mathrm{f})$, and $6 \operatorname{del}(\mathrm{g})$ primary transformants. Scale bar = $100 \mu \mathrm{m}$. 
a

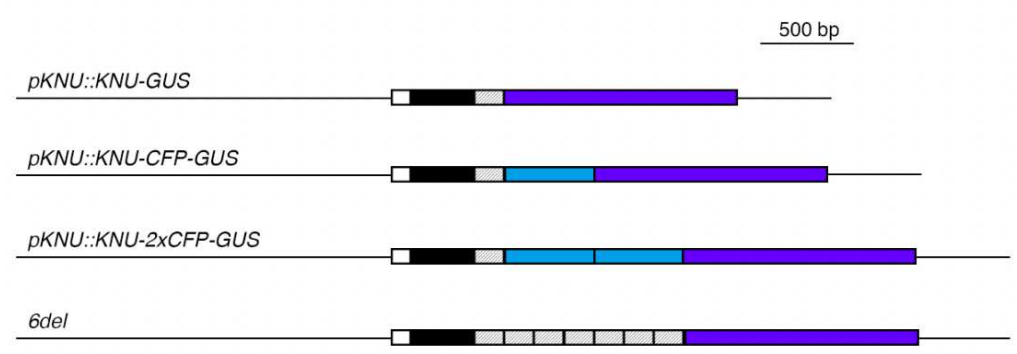

b

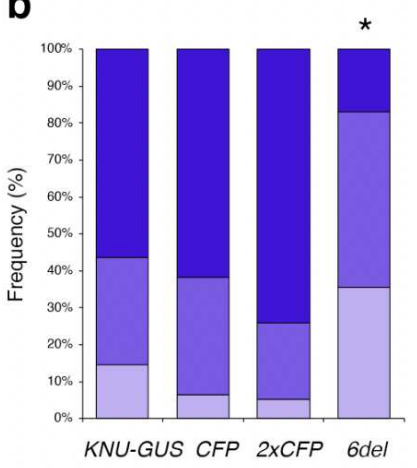

Extended Data Fig. 4. KNU fused to CFP and $2 \times$ CFP results in similar $\mathrm{T}_{1}$ GUS signal strength as $p K N U:: K N U$-GUS

a, Schematic diagrams of the $p K N U:: K N U-C F P-G U S, p K N U:: K N U-2 x C F P-G U S, p K N U:: K N U-$ GUS and $6 \mathrm{del}$ constructs. Black line, region upstream of the $K N U$ coding sequence; white bars, 5 , untranslated region (5' UTR); black bars, 255 bp of $K N U$ coding sequence; gray bars, 231-bp del sequence; light blue bars, $C F P$ or $2 x C F P$; dark blue bars, GUS. b, Variation in GUS signal strength levels in $p K N U:: K N U-C F P-G U S, p K N U:: K N U-2 x C F P-G U S, p K N U:: K N U-G U S$ and $6 d e l \mathrm{~T}_{1}$ plants. The dark blue, blue, and light blue bars represent strong, intermediate, and weak GUS signal intensity, respectively. A $X^{2}$ test was used to test for significant differences for the del plants $\left(\mathrm{n}>35,{ }^{*} p<0.05\right)$. 


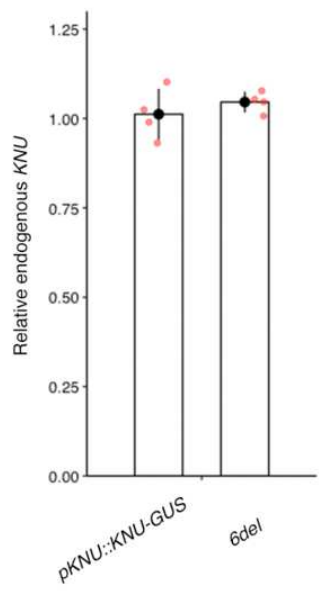

Extended Data Fig. 5. Endogenous $K N U$ mRNA levels are similar in $p K N U:: K N U$-GUS and 6 del transgenic plants

Endogenous $K N U$ transcript levels in $p K N U:: K N U$-GUS and $6 d e l$ were determined by RT-qPCR using RNA isolated from floral bud clusters containing flowers until stage 6. Data represent average fold change \pm standard errors of three independent experiments $(\mathrm{n}=4)$. TUBULIN 2 served as the reference transcript. 


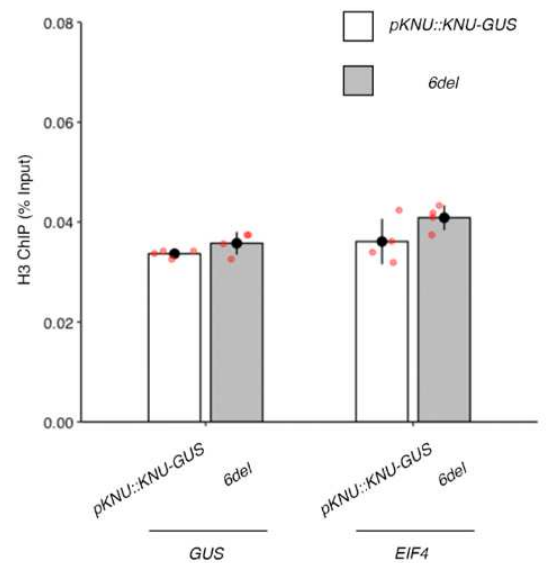

Extended Data Fig. 6. Occurrence of the histone variant $\mathrm{H3}$ is comparable in $p K N U:: K N U-G U S$ and 6 del transgenic plants

$\mathrm{H} 3$ enrichment in $p K N U:: K N U$-GUS and 6 del plants using floral bud clusters containing flowers until stage 10. Data represent average $\%$ Input \pm standard errors from three independent experiments $(\mathrm{n}=$ 4). A two-tailed Student's $t$-test was used to determine significant differences $(p<0.05)$. EIF4A1 served as the positive control. 

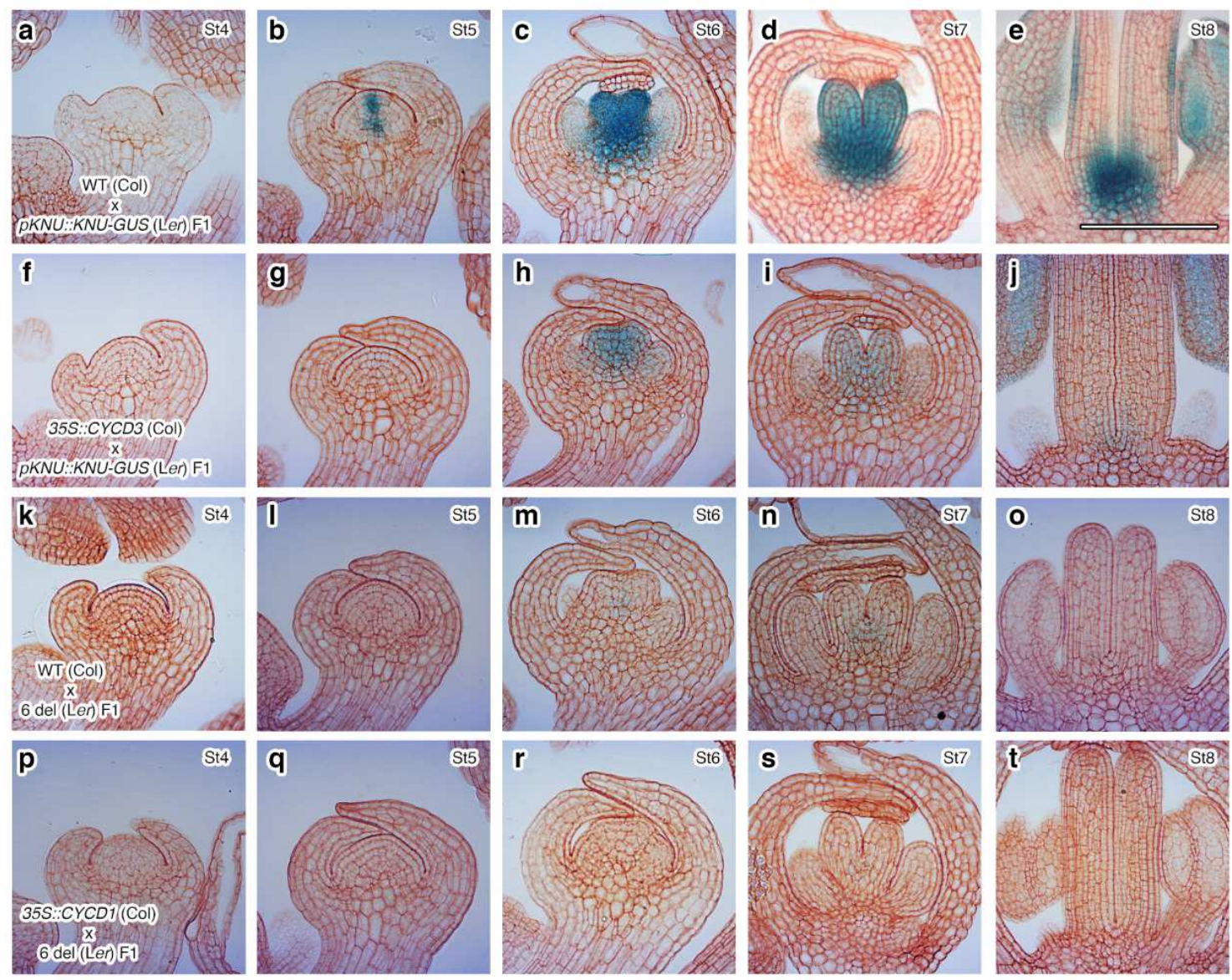

Extended Data Fig. 7. pKNU::KNU-GUS and 6del expression pattern in WT and 35S::CYCD3;1

$\mathbf{a}-\mathbf{t}$, Representative longitudinal GUS sections from stage 4-8 6 del floral buds in primary

transformants in the WT and $35 S:: C Y C D 3 ; 1$ background. Cell walls were stained with neutral red dye. Scale bar $=50 \mu \mathrm{m}$. 


\section{Supplementary Table 1. Candidates of biotimer-regulated genes}

\begin{tabular}{llll}
\hline AGI code & Gene name & Biological function & References \\
\hline$A T 1 G 62360$ & STM & Shoot apical meristem maintenance & 47 \\
$A T 4 G 02670$ & IDD12 & Cellular patterning & 52 \\
$A T 4 G 17810$ & ZP1 & Root hair development & 48 \\
$A T 5 G 14010$ & KNU & Floral meristem termination & 13 \\
$A T 4 G 16540$ & HSP20 & Flower development & 50,51 \\
$A T 3 G 11150$ & N/A & N/A & 49 \\
$A T 1 G 14600$ & N/A & N/A & 52,54 \\
$A T 3 G 60670$ & PZ10 & N/A & 53 \\
$A T 3 G 51060$ & STY1 & Gynoecium development & 24 \\
$A T 3 G 60870$ & AHL18 & Flowering & 26 \\
\hline
\end{tabular}


Supplementary Table 2. Genome-wide datasets used for in silico analysis

\begin{tabular}{lllll} 
Dataset & Genotype & Stages & Gene & References \\
& & & number \\
\hline AG-response genes & apl cal 35S::AP1-GR & $3-4$ & 245 & 23 \\
AG-response genes & apl cal 35S::AP1-GR & $5-6$ & 920 & 23 \\
AG-bound genes & ap1 cal pAP1::AP1-GR & $5-6$ & 2,224 & 23 \\
& pAG::AG-GFP & & 1,416 \\
PRC2/H3K27me3- & WT, prc2 mutants, and & \\
bound genes & tagged lines & & \\
\hline
\end{tabular}


Supplementary Table 3. Primers used in this study

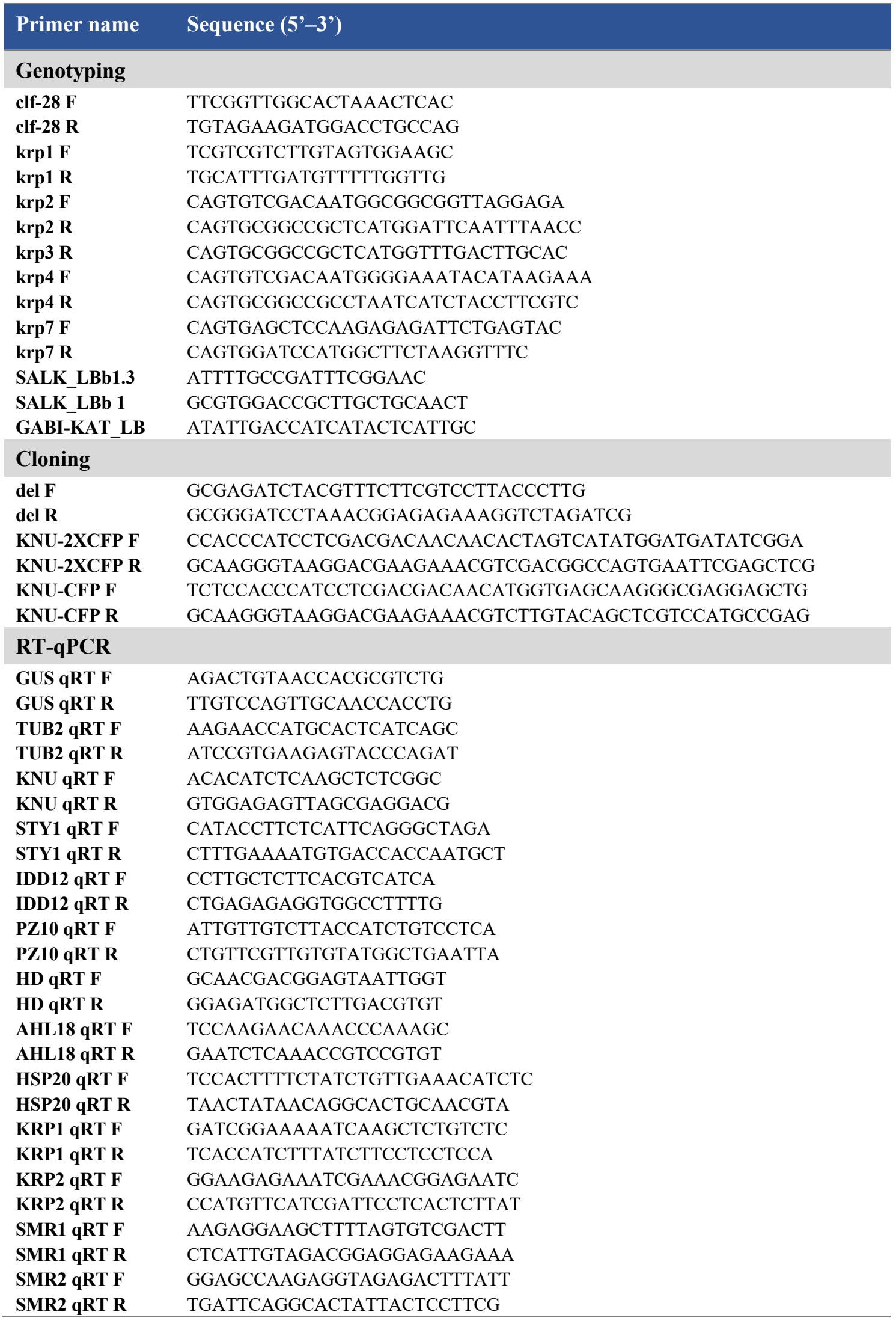




\begin{tabular}{ll}
\hline SMR3 qRT F & AGATCGAGATGTTCTTCGAGGATTT \\
SMR3 qRT R & CAAAGGATCAAATTACCACCACACA \\
ChIP-qPCR & \\
\hline KNU-GUS ChIP F & ACAACAACGACGGAGGAAAC \\
KNU-GUS $\mathbf{C h I P}$ & CCAACCCGTGAAATCAAAAAA \\
R & \\
EIF4 ChIP F & ACCAGGCGTAAGGTTGATTG \\
EIF4 ChIP R & GGTCCATGTCTCCGTGAGTT \\
KNU ChIP F & CGACGACAACAACACGTTC \\
KNU ChIP R & CATCCATCGTCATCATCGTC \\
IDD12 ChIP F & ACGCATAGAGCGTTTTGTGA \\
IDD12 ChIP R & TGGAAAGTGATGGTTCGATG \\
AHL18 ChIP F & ATGGATGAGGTATCTCGTTCTC \\
AHL18 ChIP R & AGGAAAGGTGGCTAGGTCTTC \\
\hline
\end{tabular}




\section{Supplementary Files}

This is a list of supplementary files associated with this preprint. Click to download.

- SupplementaryData1.xIsx 